\title{
Male castration alters the gut microbiota, leading to increased serum levels of branched-chain amino acids and adiposity
}

\section{Tae Woong Whon}

Kyung Hee University

Hyun Sik Kim

Kyung Hee University

Na-Ri Shin

Kyung Hee University

\section{Eun Sung Jung}

Konkuk University

\section{Euon Jung Tak}

Kyung Hee University

\section{Hojun Sung}

Kyung Hee University

Mi-Ja Jung

Kyung Hee University

\section{Yun-Seok Jeong}

Kyung Hee University

\section{Dong-Wook Hyun}

Kyung Hee University

Pil Soo Kim

Kyung Hee University

\section{Yu Kyung Jang}

Konkuk University

Choong Hwan Lee

Konkuk University

Jin-Woo Bae ( $\sim$ baejw@khu.ac.kr)

Kyung Hee University https://orcid.org/0000-0001-6433-5270

\section{Research}

Keywords: Hypogonadism, lleal microbiota, Peptostreptococcaceae, Branched-chain amino acids, Intramuscular fat 
Posted Date: February 12th, 2020

DOI: https://doi.org/10.21203/rs.2.23314/v1

License: (c) (i) This work is licensed under a Creative Commons Attribution 4.0 International License. Read Full License 


\section{Abstract}

Background : Testosterone deficiency is positively correlated with fat accumulation and obesity-related comorbidities, such as metabolic syndrome. Castration of young males is widely used in the cattle industry to improve meat quality. However, the mechanism linking hypogonadism and host metabolism is not clear. We aimed to evaluate the effect of male hypogonadism on the gut microbiota and serum metabolites, and the contribution of the altered microbiota to the host metabolic phenotype during hypogonadism.

Results : We used metataxonomic and metabolomic approaches to evaluate the intestinal microbiota and host metabolism in male, castrated male (CtM), and female cattle. We then used a male mouse castration model to evaluate the causative factor(s) that underpin the alteration of the intestinal microbiota and host metabolic phenotype in response to hypogonadism. After pubescence, the CtM cattle harbored distinct ileal microbiota dominated by the family Peptostreptococcaceae, and exhibited distinct serum and muscle amino acid profiles (i.e., highly abundant branched-chain amino acids), with increased extra- and intramuscular fat storage. Castration of male mice phenocopied both the intestinal microbial alterations and obese-prone metabolism observed in cattle. Antibiotic treatment and fecal microbiota transplantation experiments in a mouse model further revealed that the intestinal microbial alterations associated with hypogonadism are a key contributor to the obese phenotype in the CtM animals.

Conclusions: We demonstrated altered gut microbial profiles in the hypogonadal animals, with a negative feedback between the serum testosterone levels and the ileal abundance of Peptostreptococcaceae, and a distinct metabolic phenotype, with an enhanced amino acid metabolism. These findings suggest targeting the gut microbiota as a potential therapeutic strategy for the treatment of both hypogonadism and obesity.

\section{Background}

Beyond its contribution as the principal male sex hormone, testosterone plays a key role in metabolism and body composition. Elderly men and patients with Klinefelter's syndrome who usually suffer from testosterone deficiency, termed hypogonadism, often show an unfavorable body composition with increased fat mass and reduced muscle mass [1, 2]. Conversely, the incidence of hypogonadism is increased in obese men compared to healthy men $[3,4]$. This bidirectional relationship between obesity and male hypogonadism (meaning that primary hypogonadism promotes fat accumulation while obesity attenuates endogenous testosterone production) has been highlighted in the current obesity epidemic [5]. Several animal model studies also support this reciprocal parallelism. For example, genetically obese (leptin-deficient ob/ob) male mice exhibit hypogonadism-mediated impaired reproductive function [6, 7] and androgen receptor knock-out mice or castration models predispose to development of obesity $[8,9]$. It has been proposed that the enzymatic conversion of testosterone to estradiol by adipocyte-derived aromatase can cause hypogonadism in obese individuals $[10,11]$ and that testosterone inhibits 
differentiation of preadipocyte to adipocyte in vitro [12]. However, the mechanisms linking hypogonadism with fat accumulation are still not fully elucidated.

In the cattle industry, castration of male cattle is commonly practiced worldwide. Castration of bulls has advantages in terms of tractability and profitability. For instance, feeding male castrated bull (steer) a high-concentrate diet results in a greater increase of the subcutaneous and intramuscular fat, and a higher meat tenderness score, than those of non-castrated bull $[13,14]$. This, in turn, leads to a higher market price of the steer carcass. Modified expression patterns of the lipid metabolism genes and/or perturbed anabolic hormone status (i.e., insulin-like growth factor-1) reportedly play a role in the improved quality of the steer carcass $[15,16]$ but the underlying mechanisms are poorly understood.

The symbiotic gut microbiota plays a pivotal role in mammalian metabolism. Accumulating evidence indicates that the gut microbiota contributes to the development of obesity and associated metabolic disorders by regulating energy homeostasis $[17,18]$. Accordingly, in the present study, we aimed to determine the effect of hypogonadism following male castration on the gut microbiota, serum metabolites, and the host metabolic phenotype, and the contribution of the altered microbiota to increased adiposity in hypogonadal animal models. We used metataxonomic (i.e., amplification and sequencing of bacterial 16S rRNA genes) and metabolomic approaches to analyze the gastrointestinal (rumen, ileum, cecum, and colon), serum, and striploin muscle samples of male, castrated male (CtM), and female Korean brown cattle (Bos taurus coreanae; hereafter referred to as the Hanwoo) and Holstein Friesian cattle (the Holstein). We also used a male mouse castration model to evaluate the causative role of the alteration of the intestinal microbiota in the host metabolic phenotype in response to hypogonadism.

\section{Results}

\section{Male hypogonadism results in the alteration of rectal microbiota and elevated levels of the serum branched-chain amino acids (BCAA) in postpubescent cattle}

To evaluate the possible relationship between male hypogonadism and the intestinal microbiota in cattle, we investigated bacterial communities in the rectal contents of male $(n=5)$ and CtM Hanwoo $(n=5)$ at juvenile (mean age: $11.9 \pm 1.1$ months) and postpubescent stages (mean age: $19.3 \pm 1.5$ months). Agematched non-pregnant females $(n=5)$ were included in the comparison of rectal microbiota. All animal groups were farmed in geographically closely located cages and fed the same diet (Additional file 2: Supplementary Table S1) to minimize any cage- and diet-induced inter-individual variation of the intestinal microbiota. Body weight was not significantly affected by male castration (Fig. 1a). At the postpubescent stage, but not at the juvenile stage, the serum testosterone levels in the male Hanwoo were significantly higher than those in CtM and females (Fig. 1b and Additional file 1: Supplementary Fig. S1a; ANOVA, $p<0.001)$. The serum $17 \beta$-estradiol levels were not significantly different between groups at the postpubescent and juvenile stage (Fig. 1c and Additional file 1: Supplementary Fig. S1b). Principal coordinates analysis (PCOA) of the rectal microbiome indicated that the gut microbial communities in the 
juvenile or postpubescent males were significantly separated from the other groups (PERMANOVA, $p=$ 0.001; comparison of the male, CtM, and female animals) (Fig. 1d and Additional file 1: Supplementary Fig. S1c). The PCoA plots of the postpubescent CtM and female data grouped together, indicating that the effect of castration on the rectal microbiota became apparent after pubescence. We additionally investigated the impact of male castration on the rectal microbiota in another breed of cattle, the Holstein. Similar to the postpubescent Hanwoo, PCoA of the Holstein rectal microbiota data revealed a significant bacterial community separation according to male castration (PERMANOVA, $p=0.001$; comparison of the male, $\mathrm{CtM}$, and female animals), with a clustering of the CtM and female data (Additional file 1: Supplementary Fig. S2a).

To evaluate the effects of changes in the gut microbiota induced by male castration on the host metabolomic profile, we next analyzed the serum metabolome profiles of postpubescent cattle by using gas chromatography time-of-flight mass spectrometry (GC-TOF-MS). Similar to the clustering patterns of the cattle rectal microbiota described above, PCoA of the Hanwoo metabolome data revealed that the male and CtM datasets clustered separately (Fig. 1e; PERMANOVA, $p=0.073$; comparison of the male and CtM Hanwoo). The CtM and female datasets clustered closely together. Profiling of the serum metabolomes of the male and CtM Hanwoo revealed meaningful differences in the serum amino acid levels. Specifically, the serum levels of isoleucine and proline were significantly higher in the CtM Hanwoo than in the male Hanwoo (Fig. 1f; Mann-Whitney $U$ test, $p<0.05$ ). Of note, BCAAs (isoleucine, leucine, and valine) are responsible not only for the intramuscular lipid accumulation, by increasing the vascular fatty acid transport into the mouse skeletal muscle [19], but also for lipogenesis in the human adipocyte [20]. We accordingly quantified serum BCAA levels using an enzyme-based assay. The serum BCAA levels were significantly higher in the postpubescent CtM Hanwoo than in the male controls (Fig. 1g; MannWhitney $U$ test, $p<0.05$ ). The serum metabolite profiles of Holstein revealed that the levels of isoleucine, valine, phenylalanine, and threonine were significantly higher (Mann-Whitney $U$ test, $p<0.05$ ) in the CtM animals than in males (Additional file 1: Supplementary Fig. S2b,c). Further, an enzyme-based quantitative analysis revealed higher median serum BCAA levels in the CtM group than in the male controls, but the difference was not statistically significant (Additional file 1: Supplementary Fig. S2d).

\section{Metataxonomic analysis of different intestinal compartments reveals a marked increase in the family Peptostreptococcaceae in the ileum of CtM animals}

Although the analysis revealed differences in the rectal microbiota of the male and CtM cattle (Fig. 1), no direct link between the composition of rectal microbiota and elevated serum BCAA levels was apparent, nor the involvement of the rectal microbiota in the host amino acid metabolism (according to the rectal metabolome profiles of postpubescent cattle; Additional file 1: Supplementary Fig. S3). To reveal whether the high serum BCAA levels were a consequence of the gut microbial activity, we next extensively investigated microbial profiles in the different compartments of the gastrointestinal tract. We collected the luminal contents of the rumen, ileum, and colon from the adult male (mean age: $31.2 \pm 5.9$ months, $n$ $=10$ ) and CtM (mean age: $33.9 \pm 1.4$ months, $n=10$ ) Hanwoo. Similar to the postpubescent stage, we observed a significant difference (Mann-Whitney $U$ test, $p<0.001$ ) in the serum testosterone levels 
between the male and CtM groups at the adult stage (Fig. 2a). PCoA of the microbiota in the rumen, ileum, and colon revealed that the bacterial communities in the different segments were significantly different in the male and CtM Hanwoo (PERMANOVA, $p<0.005$ for each segment; comparison of the male and CtM animals in the rumen, ileum, and colon) (Fig. 2b). We next evaluated the gastrointestinal segment in which the bacterial communities were mostly affected by male castration. When all gastrointestinal samples were plotted together, only the ileal microbiota in the male and CtM groups were separated on both the abundance-weighted (weighted UniFrac, Fig. 2c and Additional file 1: Supplementary Fig. S4a) and -unweighted PCoA (unweighted UniFrac, Additional file 1: Supplementary Fig. S4b). Similar, analysis of the weighted UniFrac distance between the male and CtM groups revealed the largest dissimilarity value for the ileal microbiota (Fig. 2d).

We then compared the relative abundances of major taxa ( $>0.5 \%$ of the mean abundance) at the family level. The difference of the ileal microbiota in the male and CtM groups was apparent, with a marked increase in the family Peptostreptococcaceae in the CtM Hanwoo (Fig. 2e and Additional file 1: Supplementary Fig. S4c). Phylogenetic analysis of the operational taxonomic units (OTUs) assigned to the family Peptostreptococcaceae further revealed that the abundant OTUs mostly belonged to the genera Romboutsia and Paeniclostridium (Additional file 1: Supplementary Fig. S5).

To investigate the relationship between the intestinal microbiota and the resultant microbial metabolites (especially BCAAs) in detail, we subsequently analyzed metabolomic profiles of the luminal contents of the ileum, cecum, and colon of the CtM Hanwoo (Additional file 3: Supplementary Result). In the ileum, a strong positive correlation (Spearman's rank correlation analysis, $p<0.001$ ) was detected between the BCAAs and two unclassified genera belonging to the family Peptostreptococcaceae, and the genera Butyrivibrio, Acetitomaculum, and Atopobium (Additional file 1: Supplementary Fig. S6a). Furthermore, the ileal levels of intestinal BCAAs were significantly higher (Mann-Whitney $U$ test, $p<0.001$ ) in the CtM Hanwoo than those in the male controls; the BCAA levels in the cecum and colon were much lower than those in the ileum, and no quantitative difference in the BCAA levels was observed between the male and CtM groups (Additional file 1: Supplementary Fig. S6b).

\section{Male castration results in increased intramuscular fat accumulation with high BCAA levels in the adult cattle}

We next evaluated the consequence of the distinct metabolomic profile (i.e., high levels of BCAAs in the serum and ileum) of the CtM cattle. After the bodies were dressed ( $n=10$ in each group), we observed clear differences in the body composition of the male and CtM Hanwoo (Fig. 3a). The thickness of the dorsal subcutaneous fat, total fat weight, and serum BCAA levels in the CtM group were significantly higher than those of the male controls (Fig. 3b; Mann-Whitney $U$ test, $p<0.001$ ). We then analyzed the degree of intramuscular fat accumulation in the fresh striploin muscle in the male and CtM Hanwoo carcasses ( $n=5$ in each group). We observed a significantly higher (Mann-Whitney $U$ test, $p<0.01$ ) intramuscular fat accumulation in the CtM muscle than in the male muscle (Fig. 3c,d). We next evaluated the intramuscular metabolites profiles. Similar to the serum metabolite profiles described above, the 
intramuscular metabolomes of the male and CtM carcasses clustered significantly separately by PCoA (Fig. 3e; PERMANOVA, $p=0.008$; comparison of the male and CtM Hanwoo). The levels of BCAAs (i.e., isoleucine and leucine), as well as phenylalanine, tryptophan, and tyrosine, were significantly higher (Mann-Whitney $\mathrm{U}$ test, $\mathrm{p}<0.05$ ) in the CtM muscle than in the male muscle (Fig. 3f). Among the amino acids whose levels were increased in the CtM muscle, leucine, phenylalanine, tryptophan, and tyrosine can be degraded into acetyl-CoA, a precursor of ketone bodies. We subsequently measured the intramuscular levels of $\beta$-hydroxybutyrate (3-HB), widely used to diagnose ketosis in dairy cow [21], in the male and CtM muscles. The 3-HB levels were significantly higher (Mann-Whitney $U$ test, $p<0.01$ ) in the CtM muscle than in the male muscle (Fig. 3g). Interestingly, Jang et al. [19] reported that 3-HB secreted by the muscle cell activates endothelial fatty acid transport and promotes lipid accumulation in the muscle. This supports the data presented herein, bridging the high levels of serum BCAAs and the elevated intramuscular fat accumulation in the CtM cattle.

\section{Male hypogonadism leads to an ileal microbial alteration, adiposity, and increased serum BCAA levels in mice}

The correlation-based analysis in cattle presented above suggested an obesogenic effect(s) of the castration of young male on the subsequent host metabolic phenotype in association with altered intestinal microbiota and systemic metabolite levels. We next evaluated the causative role of the alteration of the gut microbiota following hypogonadism in the obese metabolic phenotype in a mouse model. Male mice underwent prepubertal castration by orchiectomy and were fed a high-fat diet (HFD) to promote the obese phenotype. Age-matched male mice that underwent sham-operation and fed low-fat diet (LFD) were included as the castration and diet controls, respectively ( $n=6$ in each group; Fig. 4a). At the end of the experiment, the serum testosterone levels in the CtM groups were significantly lower than those in the Sham groups (unpaired Student's t-test, $p<0.05$ ), confirming that hypogonadism developed after castration (Fig. 4b). Further, serum testosterone levels in diet-induced obese mice were lower than those in the lean controls (unpaired Student's t-test, $p=0.064$; comparison of the Sham-LFD and ShamHFD groups). This was in agreement with the results of human epidemiological studies [5]. Weighted PCoA of both the ileal and colonic microbiota revealed a male castration-associated separation of the intestinal microbial community structures, regardless of the intestinal compartment analyzed (PERMANOVA, $p<0.04$; comparison of the Sham and CtM groups). However, the difference between the ileal microbiota was more obvious than that between the colonic microbiota in HFD-fed mice (Fig. 4c and Additional file 1: Supplementary Fig. S7a,b). In both LFD- and HFD-fed mice, male hypogonadism amplified the expansion of the family Peptostreptococcaceae in the ileum (Fig. 4d and Additional file 1: Supplementary Fig. S8a,b), and resulted in significantly increased levels of serum BCAAs (Fig. 4e; unpaired Student's t-test, $\mathrm{p}<0.05$ ).

To reveal the effect(s) of gut microbiota on the host metabolism, the HFD-fed groups received a broadspectrum antibiotic cocktail in the drinking water for 5 weeks (HFD + ABX, Fig. 4a). No signs of dehydration or anorexia were observed during the antibiotic treatment. The antibiotic treatment disrupted both the ileal and colonic microbial structures (Fig. 4c and Additional file 1: Supplementary Fig. S7c and 
S8c). As expected, the antibiotic treatment abolished the previously observed increase in the serum BCAA levels in the CtM groups compared with the Sham groups (Fig. 4e). The proportions of both the extramuscular white adipose tissue depots (the posterior subcutaneous fat, mesenteric fat, and retroperitoneal fat) and intermuscular fat (located between the gastrocnemius and rectus femoris) were significantly increased by HFD feeding and castration (unpaired Student's t-test, $p<0.01$; Fig. $4 f-h$ and Additional file 1: Supplementary Fig. S9). Although the castration-induced adiposity in the HFD-fed mice was significant in antibiotic-treated groups (Sham-ABX vs. CtM-ABX), the antibiotic treatment attenuated the level of significance.

\section{Fecal microbiota transplantation (FMT) from the hypogonadal male mice donors leads to increased fat accumulation in the recipient eugonadal male mice}

To determine whether the gut microbiota was responsible for the obese phenotype in castrated mice, we performed FMT from the HFD-fed Sham or CtM donor mice to age-matched surgery-naïve eugonadal male recipient mice (Sham-R and CtM-R, respectively; $n=5$ in each group) (Fig. $5 a$ ). Intriguingly, the difference in serum testosterone levels in the Sham-R and CtM-R groups approached statistical significance (Fig. 5b; unpaired Student's t-test, $p=0.0556$ ), suggesting a bidirectional regulatory relationship between the gut microbiota and androgenic hormones. FMT resulted in the segregation of the ileal and colonic microbiota of the recipient mice according to treatment (PERMANOVA, $p \leq 0.05$; comparison of the Sham-R and CtM-R groups) (Fig. 5c and Additional file 1: Supplementary Fig. S10a). The LEfSe analysis of the ileal microbiota revealed the family Peptostreptococcaceae as the most discriminant taxon in comparisons of the Sham-R and CtM-R groups (Fig. 5d,e). Importantly, CtM-R mice recapitulated the phenotype observed in the CtM-HFD donor mice in terms of a significantly increased fat accumulation in the posterior subcutaneous, epididymal, mesenteric, and retroperitoneal depots, as well as of the intermuscular fat in the CtM-R group compared with the Sham-R group (Fig. $5 f-h$ and Additional file 1: Supplementary Fig. S10b-g; unpaired Student's t-test, $p<0.05$ ). Collectively, these observations suggest that the hypogonadism-mediated gut microbial changes contribute to the obeseprone metabolic phenotype per se. In terms of the gut-brain axis, the microbial shift in CtM-R mice toward that in CtM mice might decrease serum testosterone levels by regulating a negative feedback loop between gonadotropin-releasing hormone in the hypothalamus, luteinizing hormone/follicle-stimulating hormone in the pituitary, and testosterone in the testis.

\section{Feeding BCAA-enriched diet elicits an obese phenotype in male mice}

To evaluate whether the surplus BCAAs are the key factor contributing to the obese phenotype of the castrated animal, we fed non-castrated male mice with HFD (0\% BCAA group) or customized isocaloric BCAA-HFDs (3\% and 5\% BCAA groups) for 8 weeks (Fig. 6a). For the BCAA-HFD, casein in HFD was substituted by BCAAs (Additional file 2: Supplementary Table S2). Feeding BCAA-HFD increased the body weight gain to a greater extent than feeding HFD, with a meaningful difference after 3 weeks of feeding (Fig. 6b; unpaired Student's t-test, $p<0.05$ ). At the end of the experiment, we observed a significantly greater adiposity (characterized by the increased percentage to body weight ratio of the posterior 
subcutaneous fat, epididymal fat, mesenteric fat, and retroperitoneal fat) in the BCAA-HFD-fed groups compared with the HFD-fed group (Fig. 6c,d and Additional file 1: Supplementary Fig. S11a-h; unpaired Student's t-test, $p<0.01)$. Further, the serum BCAA levels were significantly increased in the mice fed diets containing over $3 \%$ of BCAAs, confirming systemic circulation of the dietary BCAAs (Fig. 6e; unpaired Student's t-test, $p<0.01$ ). We subsequently investigated the effect of BCAA supplementations on the gut microbiota by analyzing the ileal and colonic bacterial communities. However, no connecting evidence was apparent linking the BCAA supplementation and gut microbial alteration (Fig. $6 \mathrm{f}$ and Additional file 1: Supplementary Fig. S11i). These observations implied that the surplus BCAAs detected in the hypogonadal animals might be a consequence of the altered gut microbial activity following male castration.

\section{Hypogonadal male animals exhibit a high intestinal microbial urease activity}

Testosterone acts not only as the major androgenic hormone but also as a metabolic hormone, affecting the hepatic urea cycle to regulate the whole-body protein catabolism [22, 23]. Because the metataxonomic analyses of the ileal microbiota of castrated animals revealed a high abundance of the family Peptostreptococcaceae, we hypothesized that a positive feedback loop might exist between the hepatic urea cycle and ureolysis undertaken by the abundant gut microbiota. Accordingly, we assessed the systemic urea/ammonia metabolism activities in cattle and mice in response to male hypogonadism. In adult Hanwoo, the microbial urease activity in both the rumen and ileum in the CtM group was significantly higher (Additional file 1: Supplementary Fig. S12a; Mann-Whitney U test, $p<0.01$ ) than that in the male group. The intestinal microbial ureases hydrolyze urea to ammonia [24]. Hence, we subsequently measured the intestinal ammonia levels. The ileal ammonia levels in the CtM group were significantly higher (Additional file 1: Supplementary Fig. S12b; Mann-Whitney U test, $p<0.05$ ) than those in the male group. In the gut, ammonia produced by ureolytic bacteria is absorbed into the bloodstream and used to fuel hepatic ureagenesis [25]. Accordingly, we determined ammonia levels in the mouse serum. For the HFD-fed mice, the serum ammonia levels in the CtM group were significantly higher than those in the Sham control (Additional file 1: Supplementary Fig. S12d; unpaired Student's t-test, $p<0.05$ ). In both cattle and mice, however, no differences in the serum urea levels in the male and CtM groups were apparent (Additional file 1: Supplementary Fig. S12c,e). This was probably because of a simple diffusion of the systemic urea into the gastrointestinal tract [26]. Collectively, the above observations suggested that testosterone deficiency in the castrated animals might positively regulate the hepatic ureagenesis, and that urea (and its hydrolysis products, i.e., ammonia and carbon dioxide) potentially affects the ileal microbial profile in the CtM animals.

\section{Discussion}

Although reciprocal parallelism between obesity and hypogonadism in men has been reported by a number of cross-sectional studies, the mechanisms linking testosterone and the obese phenotype are not fully understood. Considering the obesity epidemic, the prevalence of hypogonadism is expected to increase in the coming decades, highlighting the importance of understanding these mechanisms. For the livestock, however, animal welfare, and economical feeding and management must be considered in 
equal measure. In this context, understanding the metabolic features that could potentially influence the metabolism of beef cattle is important in breeding animals representing the "metabolically healthy obese" phenotype [27] in terms of the production of high-quality animal products. Keeping these bimodal aspects in mind, we investigated the effect of male castration on the intestinal microbiota and host metabolism in cattle and mice. We demonstrated (i) altered gut microbial profiles in the CtM animals, with a negative feedback between the serum testosterone levels and the ileal abundance of Peptostreptococcaceae, and (ii) a distinct metabolomic signature of the CtM animals with an enhanced amino acid metabolism. Furthermore, (iii) the obese phenotype associated with hypogonadism was transferrable via FMT.

We used a mouse model to examine whether alterations in the gut microbiota following hypogonadism play a causative role in the obese metabolic phenotype. Generally speaking, the mouse is a monogastric animal; as such, the digestive processes and subsequent energy metabolism are different from those in ruminant animals. Importantly, however, we found a marked increase in the ileal abundance of Peptostreptococcaceae in the CtM groups of both cattle and mice, suggesting a possible link between male hypogonadism and gut microbial perturbation in other mammal species. In humans, chemical and surgical castration of the testes is used as an androgen deprivation therapy (ADT) to treat patients suffering from advanced prostate cancer [28]. Recent accumulating evidence points out that an increased level of cholesterol post-ADT is responsible for increased de novo androgen synthesis by tumor cells, which contributes to recurrence of castration-resistant prostate cancer [29]. The mechanism underlying ADT and further increases cholesterol levels is unclear. Intriguingly, Tsoi et al. [30] report that Peptostreptococcus anaerobius can trigger intracellular cholesterol biosynthesis in mouse colon cells. Further studies are needed to examine the hormonal perturbation-gut microbiota-cancer axis in detail.

The metabolic profiles of different compartments of the intestinal tract of the CtM Hanwoo indicated that the amino acid metabolism exclusively occurs in the small intestine (Additional file 1: Supplementary Fig. S6). Amino acids that are abundant in the ileum might be processed in several ways, including absorption by the small intestinal epithelial cells to produce amino acid metabolites, and/or digestion by the colonic microbiota [31]. Relatively high levels of amino acids were also detected in the serum and striploin muscle (but not in the large intestine) of the CtM cattle, suggesting that the fate of the ileal amino acids in the CtM cattle may mostly be weighted towards the small intestinal absorption. Particularly, in the current study, we demonstrated high serum BCAA levels in the castrated cattle and mice (Fig. $3 \mathrm{~b}$ and $4 \mathrm{e}$ ). Furthermore, disruption of the gut microbiota abolished the effect of castration on BCAA levels in mice, indicating that the increased circulating BCAA levels can be attributed to altered gut microbial activity rather than hypogonadism itself. Furthermore, chronic feeding of the BCAA-enriched HFD to male mice significantly increased its body weight and adiposity, and exacerbated glucose intolerance and insulin resistance (Fig. 6d and Additional file 1: Supplementary Fig. 11a-c). BCAAs are important substrates of the anaplerotic and ketogenic metabolism in various tissues, playing substantial roles in the host metabolism and subsequent metabolic disorders (i.e., insulin resistance) [32]. Pedersen et al. [33] reported a strong association between increased levels of the serum BCAAs and insulin resistance in non-diabetic human subjects. They also noted that specific members of the intestinal 
microbiota (e.g., Prevotella copri) could increase the biosynthesis of BCAAs and elicit metabolic disorders, such as insulin resistance and/or glucose intolerance. Indeed, gut-dwelling bacteria produce highly active proteases and peptidases, enabling the hydrolysis of alimentary and/or endogenous proteins, and a subsequent absorption of amino acids in the gastrointestinal tract [34]. GC-TOF-MS analysis in the cattle study revealed variations in the amounts of individual BCAAs (e.g., isoleucine, leucine, and valine) in intestinal luminal, serum, and striploin muscle samples from the male and CtM groups. However, the study was limited because we used a total BCAA detection kit for verification. In the mouse experiments, BCAA-enriched diets comprised a mixture of isoleucine, leucine, and valine. Further studies will evaluate the properties and effects of each individual BCAA on host metabolism with respect to therapeutic and management purposes in humans and breeding animals, respectively.

The high levels of global BCAAs in cattle and mice might be attributed to high production of BCAAs by gut microbiota. However, we were not able to obtain clear evidence of causation to bridge the gap between high BCAA levels and the marked increase in microbial taxa (i.e., Peptostreptococcaceae) in CtM animals. Rather, a high rate of protein breakdown may contribute to increased levels of circulating amino acids, including BCAAs. Testosterone, as a metabolic hormone, affects whole-body anabolism by negatively regulating the hepatic urea cycle $[23,35]$. The opposite is also true, as testosterone deficiency may lead to increased hepatic ureagenesis, resulting in whole-body nitrogen loss. Indeed, several lines of evidence (i.e., increased serum ammonia levels and intestinal ammonia and urease activity) support the notion of increased hepatic ureagenesis in CtM animals (Additional file 1: Supplementary Fig. S12). Mammals cannot hydrolyze urea but urease-producing bacteria cleave urea into ammonia in the gut [36]. From the perspective of the hologenome theory (namely, that the host and all of its associated microbiota form a unit of selection during the evolutionary change) [37], the ileal microbial alteration and enhanced microbial urease activity can both conceivably be used to cope with nitrogen loss. Wiebler et al. [38] described the role of an enhanced gut microbial urea hydrolysis in the urea-nitrogen recycling in a hibernating frog. Metataxonomic analysis presented in the current study revealed the presence of abundant sequences assigned to the genera Romboutsia and Paeniclostridium in the ileal samples of the CtM Hanwoo (Fig. 2e and Additional file 1: Supplementary Fig. S5). Interestingly, recent genomic analyses of species belonging to the genera Romboutsia (e.g., R. ilealis $\mathrm{CRIB}^{\top}$ ) and Paeniclostridium (e.g., P. sordellii ATCC $9714^{\top}$ ) revealed the presence of a highly conserved urease gene cluster in the respective genomes [39, 40]; this suggests that these two bacterial taxa can utilize urea as a nitrogen source. The metataxonomic data reveal the gut microbial communities in cattle and mice at the genus level of taxonomic resolution. Given that different microbial species within a genus have different metabolic functions, isolation of key species from the gut of CtM animals to evaluate the effects of microbial signatures for host metabolism will be of interest. In addition, several sex steroid hormones regulate the secretion of digestive enzymes by the pancreas to the small intestine [41]. In a rodent, an impaired pancreatic secretion (i.e., antimicrobial peptide secretion to the small intestine) affects the intestinal microbiota and, consequently, the fitness of the host, e.g., the weight and survival rate [42]. The results of the current study, together with those of recently published studies, warrant further investigation to identify the mechanism linking hypogonadism and alteration of the intestinal microbiota.

Page $11 / 30$ 


\section{Conclusions}

Taken together, the observations presented herein indicate that primary hypogonadism as an outcome of castration leads to alterations in the ileal microbiota (i.e., dominance of the family

Peptostreptococcaceae); changes of the systemic, muscular, and intestinal metabolome profiles (i.e., high abundance of BCAAs); and increased fat storage in the cattle and mice. We also uncovered bidirectional regulation of the circulating testosterone levels by the gut microbiota as revealed by the FMT experiment. These findings suggest that hypogonadism may integrate microbiota-derived cues to modulate the host metabolism, suggesting that targeting the gut microbiota could constitute a potential therapeutic approach for the treatment of hypogonadism, as well as the associated metabolic disorders.

\section{Methods}

\section{Cattle studies}

To obtain the CtM animals, male cattle were orchiectomized by a veterinarian (both testicles were removed). Prior to sample collection, the cattle were examined for symptoms of diarrhea, and antibiotic and other medication treatment within 1 month of sampling. Rectal material and the blood were sampled from 45 healthy animals: juvenile (male, CtM, and female; mean age: $11.9 \pm 1.1$ months; $n=5$ of each) and postpubescent (male, CtM, and female; mean age: $19.3 \pm 1.5$ months; $\mathrm{n}=5$ of each) Hanwoo, and postpubescent (male, CtM, and female; mean age: $12.1 \pm 1.6$ months; $n=5$ of each) Holsteins. The rectal luminal content was collected by rectal enema using clean disposable latex gloves. The blood samples $(5 \mathrm{ml})$ were collected from the jugular vein by a veterinarian and immediately centrifuged in BD Microtainer chemistry tubes (Becton Dickinson, Franklin Lakes, NJ, USA) for serum collection. The blood and muscle samples, and the luminal content of the rumen, ileum, cecum, and colon of the adult male (mean age: $31.2 \pm 5.9$ months) and CtM Hanwoo (mean age: $33.9 \pm 1.4$ months, $n=10$ in each group) were collected from a local slaughterhouse (Gunwi-Gun, South Korea) under the supervision of the official veterinarian; the serum was collected from whole-blood samples as described above. The collected samples were transported to the laboratory on dry ice and stored at $-80^{\circ} \mathrm{C}$ until use.

\section{Mouse studies}

C57BL/6J mice were purchased from the Japan SLC Inc. (Hamamatsu, Japan) and supplied by Central Lab. Animal Inc. (Seoul, South Korea). All mice were housed in individually ventilated cages in a temperature-controlled room with a $12 \mathrm{~h}$ light/12 $\mathrm{h}$ dark cycle, and given sterilized bedding and food and water ad libitum. At the end of the experiments, mice were fasted $(6 \mathrm{~h})$, anesthetized by isoflurane inhalation, and euthanized by cardiac exsanguination. To sample the intestinal (luminal) contents, the target regions of the intestinal tract were flushed with sterile phosphate-buffered saline (PBS).

\section{Castrated male mouse studies}

To obtain the CtM animals, 4-week-old male mice were castrated by orchiectomy. For the Sham groups, age-matched male mice underwent a sham operation (i.e., the muscle layer and skin incision). After the 
surgery, both groups were given antibiotics (enrofloxacin) and an analgesic (Repellent). To reverse the effect of enrofloxacin on the gut microbiota, the Sham and CtM mice underwent FMT (the fecal samples were collected from the strain-, age-, and sex-matched non-operated donors, $n=10$ ) once a day for 2 continuous days prior to the dietary intervention. Subsequently, the mice were provided either a LFD (10\% fat; D12450H, Research Diets, New Brunswick, NJ, USA) or a HFD (45\% fat; D12451, Research Diet). For the antibiotic-treated groups, the HFD-fed mice were given a combination of ampicillin $(1 \mathrm{~g} / \mathrm{l})$, neomycin $(1 \mathrm{~g} / \mathrm{l})$, metronidazole $(1 \mathrm{~g} / \mathrm{l})$, and vancomycin $(0.5 \mathrm{~g} / \mathrm{l})$ in drinking water for 5 weeks $(\mathrm{n}=6$ per group; Fig. 4a). The antibiotic cocktail was renewed every $4 \mathrm{~d}$.

\section{Mouse FMT studies}

Non-castrated male mice (6-week-old) were maintained on LFD for 10 weeks. Prior to FMT, the mice received an antibiotic cocktail in drinking water. After a $12 \mathrm{~h}$ washout period, the mice were subjected to FMT (once a day for 3 continuous days) and fed HFD for 4 weeks. Fecal pellets from the Sham-HFD and CtM-HFD mouse donors ( $\mathrm{n}=6$ in each group) were freshly collected and transferred immediately to an anaerobic chamber (Bactron II-2, Sheldon Manufacturing) filled with an atmosphere of $5 \% \mathrm{H}_{2}, 5 \% \mathrm{CO}_{2}$, and $90 \% \mathrm{~N}_{2}$. The pellets were resuspended in sterile and reduced PBS (100 $\mathrm{mg}$ feces/ml PBS), vortexmixed for $3 \mathrm{~min}$, and sieved through a $70 \propto \mathrm{m}$ cell strainer. The fecal suspension (inoculum) was kept in Hungate anaerobic culture tubes and administered to the recipient mice (Sham-R and CtM-R, $n=5$ in each group; Fig. 5a).

BCAA-enriched diet feeding studies

Non-castrated male mice (7-week-old) were acclimated on HFD for 1 week. The mice were fed HFD ( $0 \%$ BCAA) or a customized isocaloric BCAA-HFD ( $3 \%$ and $5 \%$ BCAA) for 8 weeks ( $n=6$ in each group; Fig. 6a). The amount of each BCAA in the BCAA-enriched HFD is set out in Additional file 2: Supplementary Table S2.

\section{DNA extraction and 16S rRNA gene sequencing}

Bacterial genomic DNA was extracted from the intestinal luminal content of cattle and mice using a method involving the Repeated Bead-Beating plus column (QIAamp DNA stool mini kit; Qiagen, Valencia, CA, USA).[43] In preparation for the GS-FLX sequencing of the rectal samples from the juvenile and postpubescent cattle, a fragment of the $16 \mathrm{~S}$ rRNA gene spanning the hypervariable V1-V2 regions was amplified by polymerase chain reaction (PCR) using bacterial universal primers 8F (5'-X-GAG TTT GAT CMT GGC TCA G-3') and 338R (5'-TGC TGC CTC CCG TAG GAG T-3'); 10 b sample-specific barcodes (designated as $\mathrm{X}$ ) were added to the 5 ' primer terminus to tag the PCR products. In preparation for the Illumina MiSeq sequencing of the gastrointestinal samples from the adult Hanwoo and mice, a fragment of the 16S rRNA gene spanning the hypervariable V3-V4 regions was amplified by PCR using the forward primer 5'-TCG TCG GCA GCG TCA GAT GTG TAT AAG AGA CAG CCT ACG GGN GGC WGC AG-3' and the reverse primer 5'-GTC TCG TGG GCT CGG AGA TGT GTA TAA GAG ACA GGA CTA CHV GGG TAT CTA ATC C-3'. The PCR was performed in a C 1000 thermal cycler (Bio-Rad, Hercules, CA, USA). The PCR conditions were as follows: initial denaturation at $96^{\circ} \mathrm{C}$ for $6 \mathrm{~min}$; followed by repeated cycles of denaturation at $94{ }^{\circ} \mathrm{C}$ for $1 \mathrm{~min}$, annealing at $50^{\circ} \mathrm{C}$ for $30 \mathrm{~s}$, and extension at $72{ }^{\circ} \mathrm{C}$ for $90 \mathrm{~s}$; and a final extension step at 
$72{ }^{\circ} \mathrm{C}$ for $10 \mathrm{~min}$. For the PCR of DNA extracted from the rectum, rumen, cecum, and colon contents, 18 repeated cycles were used; for the PCR of DNA extracted from the ileum content, 25 repeated cycles were used. Three PCR reactions with the same template were pooled and the products were purified using the QIAquick PCR purification kit (Qiagen). Equimolar amounts of the purified amplicons were then combined in a single tube. For the GS-FLX sequencing, the amplicons were pyrosequenced by Macrogen (Seoul, Republic of Korea) using the Genome Sequencer FLX Titanium (Roche, Branford, CT, USA). For the Illumina MiSeq sequencing, a 16S V3-4 PCR product library was prepared using the Nextera XT Index (Illumina). The library was then sequenced using an Illumina MiSeq platform and the paired-end 2 . 300 bp reagent kit, according to the manufacturer's instructions.

\section{Evaluation of contaminating DNA}

Possible DNA contamination of all reagents used for DNA extraction and sequencing was investigated. PCR analysis for the presence of contaminating bacterial 16S rRNA genes (in a 30 cycle reaction) revealed no apparent contamination of any reagent used.

\section{Sequence analysis}

To generate the GS-FLX sequencing datasets, the raw sequences were preprocessed using the QIIME software package 1.9.0 [44] to exclude poor-quality sequences and/or sequencing errors. Briefly, raw sequences containing more than one ambiguous base call, with errors in the barcode or primer regions, with average quality scores $<25$, or shorter than $200 \mathrm{bp}$ were removed. To generate the Illumina MiSeq sequencing datasets, paired-end reads (forward and reverse fastq files) were joined and quality-filtered using the QIIME software. Chimeric sequences were excluded from the quality-filtered sequence sets using USEARCH software (version 7.0.1090). Unless noted otherwise in the text, default parameters were used. UCLUST software (version 1.2.21) clustered OTUs (at $97 \%$ sequence similarity) using the open reference OTU picking method. Singleton OTUs were excluded from further analyses. A representative sequence was selected for each OTU and aligned with reference sequences deposited in the Greengenes database using PyNAST (version 1.2.2). A phylogenetic tree of the aligned sequences was then constructed using Fasttree. Overviews of the 16S rRNA gene sequence datasets are provided in supplemental tables, as follows: the GS-FLX-generated datasets: the juvenile Hanwoo (15 samples) in Additional file 2: Supplementary Table S3; postpubescent Hanwoo (15 samples) in Additional file 2: Supplementary Table S4; and postpubescent Holstein (15 samples) in Additional file 2: Supplementary Table S5. The Illumina MiSeq-generated datasets: the adult Hanwoo (60 samples) in Additional file 2: Supplementary Table S6; castrated or sham-operated male mice (72 samples) in Additional file 2: Supplementary Table S7; FMT recipient mice (22 samples) in Additional file 2: Supplementary Table S8; and BCAA-HFD-fed mice (36 samples) in Additional file 2: Supplementary Table S9.

For phylogenetic analysis of abundant OTUs assigned to the family Peptostreptococcaceae, representative sequences were aligned with those of closely related species using the multiple sequence alignment program CLUSTAL W (Bioedit 7.2.5) [45]. A phylogenetic consensus tree was reconstructed 
using MEGA version 7 [46] and the neighbor-joining algorithm [47] based on 1000 randomly chosen bootstrap replications.

\section{Metabolite extraction}

Both extracellular and intracellular extracts of intestinal luminal contents, serum, and muscle were prepared for metabolite profiling as follows: the luminal contents $(250-500 \mathrm{mg})$ of the ileum, cecum, and colon were extracted into $1 \mathrm{ml}$ water using the MM400 mixer mill (Retsch ${ }^{\circledR}$, Haan, Germany) at a frequency of $30 \mathrm{~s}^{-1}$ for $5 \mathrm{~min}$. After sonication (10 min) and centrifugation $\left(12,578 \cdot \mathrm{g}, 4^{\circ} \mathrm{C}, 10 \mathrm{~min}\right)$, the supernatant was filtered through a $0.2 \propto \mathrm{m}$ polytetrafluoroethylene (PTFE) filter and concentrated using a speed-vacuum concentrator. For the serum extractions, $1 \mathrm{ml}$ methanol was added to $200 \propto \mathrm{l}$ of the serum, and then extracted by sonication and shaking for $10 \mathrm{~min}$. After centrifugation $\left(12,000 \mathrm{rpm}, 4^{\circ} \mathrm{C}, 10 \mathrm{~min}\right)$, the supernatant was filtered through a $0.2 \propto \mathrm{m}$ PTFE filter and dried using a speed-vacuum concentrator. The muscle samples $(5 \mathrm{~g})$ were finely chopped before extraction, following which $10 \mathrm{ml}$ methanol was added; the samples were then sonicated $(10 \mathrm{~min})$ and agitated $(1 \mathrm{~h})$. After centrifugation $(12,000 \mathrm{rpm}$, $4{ }^{\circ} \mathrm{C}, 10 \mathrm{~min}$ ), the supernatant was filtered through a $0.2 \propto \mathrm{m}$ PTFE filter and dried using a speed-vacuum concentrator.

\section{Metabolite profiling and data processing}

The dried samples were oximated in $50 \propto$ l methoxyamine hydrochloride $(20 \mathrm{mg} / \mathrm{ml}$ in pyridine) for $90 \mathrm{~min}$ at $30{ }^{\circ} \mathrm{C}$, silylated in $50 \propto \mid \mathrm{N}$-methyl-N-(trimethylsilyl) trifluoroacetamide for $30 \mathrm{~min}$ at $37^{\circ} \mathrm{C}$, and analyzed by GC-TOF-MS, as previously described [48]. The analysis was performed using an Agilent 7890 gas chromatography system (Agilent Technologies, Palo Alto, CA, USA), an Agilent 7693 auto-sampler (Agilent Technologies), and a Pegasus HT TOF MS (LECO, St. Joseph, MI, USA) system.

The GC-TOF-MS data were acquired and preprocessed using the LECO Chroma TOF $^{\text {TM }}$ software (version 4.44; LECO Corp., St. Joseph, MI, USA) and converted to the NetCDF format (*.cdf) using the LECO Chroma TOF ${ }^{\mathrm{TM}}$ software. After conversion, peak detection, retention time correction, and alignment were assessed using MetAlign 3.0 (http://www.metalign.nl). Data were exported to an Excel file. Multivariate statistical analyses were conducted using the SIMCA-P ${ }^{+}$program (version 12.0, Umetrics, Umea, Sweden). The datasets were auto-scaled and mean-centered in a column-wise fashion. The discriminant variables were selected based on the variable importance in projection $(\mathrm{VIP})$ value $(\mathrm{VIP}>0.7)$.

\section{BCAA quantification}

BCAA levels were determined in the serum samples from cattle and mice, and from the intestinal luminal contents from cattle. The BCAA concentrations were quantified using the BCAA assay kit (Sigma, St.

Louis, MO, USA). A standard curve was constructed for each experiment using a leucine standards series (MAK003D, Sigma). The BCAA levels were then calculated based on the standard curves.

\section{Hormone measurements}


Serum samples from cattle and mice were prepared as described above. The concentrations of serum testosterone and $17 \beta$-estradiol were determined by enzyme-linked immunosorbent assay (ELISA) using a testosterone ELISA kit (Enzo Life Sciences, Farmingdale, NY, USA) and a 17ß-estradiol high sensitivity ELISA kit (Enzo Life Sciences), respectively. Concentration of the serum insulin was determined by using a mouse ultrasensitive insulin ELISA kit (ALPCO, Salem, NH, USA).

\section{Analysis of gastrointestinal urease activity}

Urease activity was assayed in the ruminal and ileal luminal contents of the adult Hanwoo. The luminal contents were suspended in 10 volumes of PBS. The samples were homogenized in an assay buffer using a bead-beating method. After centrifugation, urease activity in the clear lysate solutions was determined by using a urease activity assay kit (MAK120, Sigma). A standard curve was constructed for each experiment using a series of ammonium chloride standards (MAK120C, Sigma). The amount of urease enzymatic activity in the samples was calculated according to the manufacturer's instructions.

\section{Ammonia and urea measurements}

The ammonia levels in the rumen and ileum of the adult Hanwoo were determined using the urease activity assay kit as described above. Ammonia levels in the mouse serum were determined using an ammonia assay kit (AA0100, Sigma), according to the manufacturer's instructions. A standard curve was constructed for each experiment using a series of ammonium sulfate standards (A0978, Sigma). To determine urea levels in the mouse serum, a urea assay kit (MAK006) was used. A standard curve for each experiment was constructed using a series of urea standards (MAK006F, Sigma).

\section{Glucose tolerance test}

Glucose tolerance test was performed using overnight ( $16 \mathrm{~h}$ )-fasted mice by an intraperitoneal injection of glucose ( $1 \mathrm{~g} / \mathrm{kg}$ body weight). Blood glucose concentrations were measured with an Accu-Check glucometer (Roche) before (0 min), and 15, 30, 60, and 120 min after glucose injection.

\section{Intramuscular fat image analysis}

Striploin muscle images were size-normalized. The sizes of intramuscular fat areas were calculated using the i-Solution image analyzer (IMT i-Solution Inc., Vancouver, BC, Canada).

\section{Statistics}

The statistical analyses were performed using GraphPad Prism version 5.0 for Windows (GraphPad Software, La Jolla, CA, USA). Comparisons between two samples were made using the non-parametric Mann-Whitney $U$ test and the unpaired Student's $t$-test (one-tailed). Comparisons between multiple samples were conducted by the analysis of variance (ANOVA), followed by Tukey's post-hoc test ( ${ }^{*} \mathrm{p}<0.05$, $* \star p<0.01$, and $* \star \star p<0.001)$. The statistical significance for the observed variations was assessed using the PERMANOVA function with 999 permutations. The lines, boxes, and whiskers in the box plot diagrams 
represent the median, first and third quartiles, and min-to-max distribution of replicate values, respectively. The values and scattered dots in the bar graphs represent the means \pm SEM and the individual replicates, respectively.

\section{Abbreviations}

ANOVA, analysis of variance;

3-HB, b-hydroxybutyrate;

BCAA, branched-chain amino acids;

CtM, castrated male;

FMT, fecal microbiota transplantation;

GC-TOF-MS, gas chromatography time-of-flight mass spectrometry;

HFD, high-fat diet;

LFD, low-fat diet;

OTUs, operational taxonomic units;

PCoA, Principal coordinates analysis.

\section{Declarations}

\section{Ethics approval}

The study protocol was approved by the Institutional Animal Care and Use Committee of Kyung Hee University [KHUASP(SE)-17-026] for cattle study and [KHUASP(SE)-18-010] for mice study, and the experiments were performed in agreement with the ARRIVE guidelines as described above.

\section{Consent for publication}

Not applicable

\section{Availability of data and materials}

The raw sequences of 16S rRNA genes obtained from the intestinal luminal content of cattle and mice have been deposited in the European Nucleotide Archive, and are available under the accession number PRJEB19502. 
The authors declare that they have no competing interests.

\section{Funding}

This study was supported by the Korea Institute of Planning and Evaluation for Technology in Food, Agriculture, and Forestry (IPET) through the Agricultural Microbiome R\&D Program, funded by the Ministry of Agriculture, Food, and Rural Affairs (MAFRA) (918011-04-1-SB010); the National Research Foundation of Korea (NRF) grant funded by the Korean government (MSIT) (grant number: NRF2018R1A5A1025077); the Mid-Career Researcher Program (NRF-2016R1E1A1A02921587); and the Bio \& Medical Technology Development Program (NRF-2017M3A9F3046549).

\section{Author contributions}

J-WB and TWW designed the experiments. TWW, HSK, and N-RS performed the majority of the experiments and analyzed the data. EJT, HS, M-JJ, Y-SJ, D-WH, and PSK helped with sample collection and data presentation. ESJ, YKJ, and CHL performed the metabolomics experiments and analyzed the data. TWW, HSK, N-RS, and J-WB wrote the manuscript.

\section{Acknowledgements}

We thank the farm owners, veterinarians, and slaughterhouse staff for helping with sample collection. We especially thank Hong Gil Kim (President of the National Hanwoo Association) and Sang Uk Han (CJ CheilJedang Corp.) for advising on the sampling strategy and helpful discussions.

\section{References}

1. Bojesen A, Kristensen K, Birkebaek NH, Fedder J, Mosekilde L, Bennett P, Laurberg P, Frystyk J, Flyvbjerg A, Christiansen JS, Gravholt $\mathrm{CH}$ : The metabolic syndrome is frequent in Klinefelter's syndrome and is associated with abdominal obesity and hypogonadism. Diabetes Care 2006, 29:1591-1598.

2. Tajar A, Forti G, O'Neill TW, Lee DM, Silman AJ, Finn JD, Bartfai G, Boonen S, Casanueva FF, Giwercman A, et al: Characteristics of secondary, primary, and compensated hypogonadism in aging men: evidence from the European Male Ageing Study. J Clin Endocrinol Metab 2010, 95:1810-1818.

3. Dhindsa S, Ghanim H, Batra M, Dandona P: Hypogonadotropic Hypogonadism in Men With Diabesity. Diabetes Care 2018, 41:1516-1525.

4. Saboor Aftab SA, Kumar S, Barber TM: The role of obesity and type 2 diabetes mellitus in the development of male obesity-associated secondary hypogonadism. Clin Endocrinol (Oxf) 2013, 78:330-337.

5. Carrageta DF, Oliveira PF, Alves MG, Monteiro MP: Obesity and male hypogonadism: Tales of a vicious cycle. Obes Rev 2019. 
6. Batt RA, Everard DM, Gillies G, Wilkinson M, Wilson CA, Yeo TA: Investigation into the hypogonadism of the obese mouse (genotype ob/ob). J Reprod Fertil 1982, 64:363-371.

7. Swerdloff RS, Batt RA, Bray GA: Reproductive hormonal function in the genetically obese (ob/ob) mouse. Endocrinology 1976, 98:1359-1364.

8. Harada N, Hanaoka R, Horiuchi H, Kitakaze T, Mitani T, Inui H, Yamaji R: Castration influences intestinal microflora and induces abdominal obesity in high-fat diet-fed mice. Sci Rep 2016, 6:23001.

9. Rana K, Fam BC, Clarke MV, Pang TP, Zajac JD, MacLean HE: Increased adiposity in DNA bindingdependent androgen receptor knockout male mice associated with decreased voluntary activity and not insulin resistance. Am J Physiol Endocrinol Metab 2011, 301:E767-778.

10. Hayes FJ, DeCruz S, Seminara SB, Boepple PA, Crowley WF, Jr.: Differential regulation of gonadotropin secretion by testosterone in the human male: absence of a negative feedback effect of testosterone on follicle-stimulating hormone secretion. J Clin Endocrinol Metab 2001, 86:53-58.

11. Hayes FJ, Seminara SB, Decruz S, Boepple PA, Crowley WF, Jr.: Aromatase inhibition in the human male reveals a hypothalamic site of estrogen feedback. J Clin Endocrinol Metab 2000, 85:3027-3035.

12. Singh R, Artaza JN, Taylor WE, Braga M, Yuan X, Gonzalez-Cadavid NF, Bhasin S: Testosterone inhibits adipogenic differentiation in 3T3-L1 cells: nuclear translocation of androgen receptor complex with beta-catenin and T-cell factor 4 may bypass canonical Wnt signaling to down-regulate adipogenic transcription factors. Endocrinology 2006, 147:141-154.

13. Marti S, Jackson JA, Slootmans N, Lopez E, Hodge A, Perez-Juan M, Devant M, Amatayakul-Chantler S: Effects on performance and meat quality of Holstein bulls fed high concentrate diets without implants following immunological castration. Meat Sci 2017, 126:36-42.

14. Field RA: Effect of castration on meat quality and quantity. J Anim Sci 1971, 32:849-858.

15. Jeong J, Bong J, Kim GD, Joo ST, Lee HJ, Baik M: Transcriptome changes favoring intramuscular fat deposition in the longissimus muscle following castration of bulls. J Anim Sci 2013, 91:4692-4704.

16. Plouzek CA, Trenkle A: Insulin-like growth factor-I concentrations in plasma of intact and castrated male and female cattle at four ages. Domest Anim Endocrinol 1991, 8:73-79.

17. Turnbaugh PJ, Ley RE, Mahowald MA, Magrini V, Mardis ER, Gordon JI: An obesity-associated gut microbiome with increased capacity for energy harvest. Nature 2006, 444:1027-1031.

18. Shin NR, Lee JC, Lee HY, Kim MS, Whon TW, Lee MS, Bae JW: An increase in the Akkermansia spp. population induced by metformin treatment improves glucose homeostasis in diet-induced obese mice. Gut 2014, 63:727-735.

19. Jang C, Oh SF, Wada S, Rowe GC, Liu L, Chan MC, Rhee J, Hoshino A, Kim B, Ibrahim A, et al: A branched-chain amino acid metabolite drives vascular fatty acid transport and causes insulin resistance. Nat Med 2016, 22:421-426.

20. Green CR, Wallace M, Divakaruni AS, Phillips SA, Murphy AN, Ciaraldi TP, Metallo CM: Branched-chain amino acid catabolism fuels adipocyte differentiation and lipogenesis. Nat Chem Bio/ 2016, 12:1521. 
21. Zhang HY, Wu L, Xu C, Xia C, Sun LW, Shu S: Plasma metabolomic profiling of dairy cows affected with ketosis using gas chromatography/mass spectrometry. Bmc Veterinary Research 2013, 9.

22. Lam T, Poljak A, McLean M, Bahl N, Ho KK, Birzniece V: Testosterone prevents protein loss via the hepatic urea cycle in human. Eur J Endocrinol 2017, 176:489-496.

23. Birzniece V: Hepatic actions of androgens in the regulation of metabolism. Curr Opin Endocrinol Diabetes Obes 2018, 25:201-208.

24. Patra AK, Aschenbach JR: Ureases in the gastrointestinal tracts of ruminant and monogastric animals and their implication in urea-N/ammonia metabolism: A review. $J$ Adv Res 2018, 13:39-50.

25. Liu J, Lkhagva E, Chung HJ, Kim HJ, Hong ST: The Pharmabiotic Approach to Treat Hyperammonemia. Nutrients 2018, 10.

26. Abdoun K, Stumpff F, Martens H: Ammonia and urea transport across the rumen epithelium: a review. Anim Health Res Rev 2006, 7:43-59.

27. Stefan N, Haring HU, Hu FB, Schulze MB: Metabolically healthy obesity: epidemiology, mechanisms, and clinical implications. Lancet Diabetes Endocrinol 2013, 1:152-162.

28. Feldman BJ, Feldman D: The development of androgen-independent prostate cancer. Nat Rev Cancer 2001, 1:34-45.

29. Twiddy AL, Leon CG, Wasan KM: Cholesterol as a Potential Target for Castration-Resistant Prostate Cancer. Pharmaceutical Research 2011, 28:423-437.

30. Tsoi H, Chu ESH, Zhang X, Sheng J, Nakatsu G, Ng SC, Chan AWH, Chan FKL, Sung JJY, Yu J: Peptostreptococcus anaerobius Induces Intracellular Cholesterol Biosynthesis in Colon Cells to Induce Proliferation and Causes Dysplasia in Mice. Gastroenterology 2017, 152:1419-1433 e1415.

31. Dai ZL, Wu ZL, Hang SQ, Zhu WY, Wu GY: Amino acid metabolism in intestinal bacteria and its potential implications for mammalian reproduction. Molecular Human Reproduction 2015, 21:389409.

32. Lynch CJ, Adams SH: Branched-chain amino acids in metabolic signalling and insulin resistance. Nature Reviews Endocrinology 2014, 10:723-736.

33. Pedersen HK, Gudmundsdottir V, Nielsen HB, Hyotylainen T, Nielsen T, Jensen BA, Forslund K, Hildebrand F, Prifti E, Falony G, et al: Human gut microbes impact host serum metabolome and insulin sensitivity. Nature 2016, 535:376-381.

34. Neis EP, Dejong CH, Rensen SS: The role of microbial amino acid metabolism in host metabolism. Nutrients 2015, 7:2930-2946.

35. Kelly DM, Jones TH: Testosterone: a metabolic hormone in health and disease. J Endocrino/ 2013, 217:R25-45.

36. Walser M, Bodenlos LJ: Urea metabolism in man. J Clin Invest 1959, 38:1617-1626.

37. Zilber-Rosenberg I, Rosenberg E: Role of microorganisms in the evolution of animals and plants: the hologenome theory of evolution. FEMS Microbiol Rev 2008, 32:723-735. 
38. Wiebler JM, Kohl KD, Lee RE, Jr., Costanzo JP: Urea hydrolysis by gut bacteria in a hibernating frog: evidence for urea-nitrogen recycling in Amphibia. Proc Biol Sci 2018, 285.

39. Gerritsen J, Hornung B, Renckens B, van Hijum S, Martins Dos Santos VAP, Rijkers GT, Schaap PJ, de Vos WM, Smidt H: Genomic and functional analysis of Romboutsia ilealis CRIB(T) reveals adaptation to the small intestine. PeerJ 2017, 5:e3698.

40. Couchman EC, Browne HP, Dunn M, Lawley TD, Songer JG, Hall V, Petrovska L, Vidor C, Awad M, Lyras D, Fairweather NF: Clostridium sordellii genome analysis reveals plasmid localized toxin genes encoded within pathogenicity loci. BMC Genomics 2015, 16:392.

41. Chen TS, Doong ML, Chang FY, Lee SD, Wang PS: Effects of sex steroid hormones on gastric emptying and gastrointestinal transit in rats. Am J Physio/ 1995, 268:G171-176.

42. Ahuja M, Schwartz DM, Tandon M, Son A, Zeng M, Swaim W, Eckhaus M, Hoffman V, Cui Y, Xiao B, et al: Orai1-Mediated Antimicrobial Secretion from Pancreatic Acini Shapes the Gut Microbiome and Regulates Gut Innate Immunity. Cell Metab 2017, 25:635-646.

43. Yu Z, Morrison M: Improved extraction of PCR-quality community DNA from digesta and fecal samples. Biotechniques 2004, 36:808-812.

44. Caporaso JG, Kuczynski J, Stombaugh J, Bittinger K, Bushman FD, Costello EK, Fierer N, Pena AG, Goodrich JK, Gordon JI, et al: QIIME allows analysis of high-throughput community sequencing data. Nat Methods 2010, 7:335-336.

45. Thompson JD, Gibson TJ, Plewniak F, Jeanmougin F, Higgins DG: The CLUSTAL_X windows interface: flexible strategies for multiple sequence alignment aided by quality analysis tools. Nucleic Acids Res 1997, 25:4876-4882.

46. Kumar S, Stecher G, Tamura K: MEGA7: Molecular Evolutionary Genetics Analysis Version 7.0 for Bigger Datasets. Mol Biol Evol 2016, 33:1870-1874.

47. Saitou N, Nei M: The neighbor-joining method: a new method for reconstructing phylogenetic trees. Mol Biol Evol 1987, 4:406-425.

48. Jung ES, Park HM, Lee KE, Shin JH, Mun S, Kim JK, Lee SJ, Liu KH, Hwang JK, Lee CH: A metabolomics approach shows that catechin-enriched green tea attenuates ultraviolet B-induced skin metabolite alterations in mice. Metabolomics 2015, 11:861-871.

\section{Additional Files}

Additional file 1: Supplementary Figures (S1-S12).

Additional file 2: Supplementary Tables (S1-S9).

Additional file 3: Supplementary Result.

\section{Figures}


a

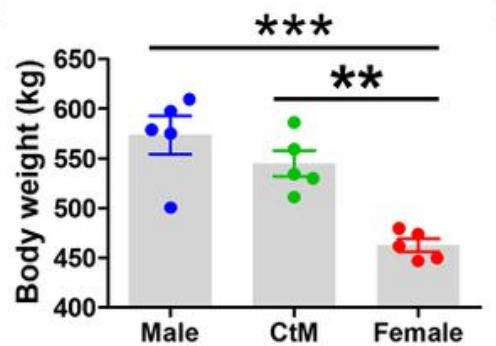

b

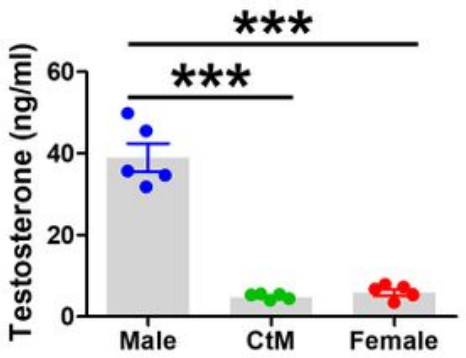

C

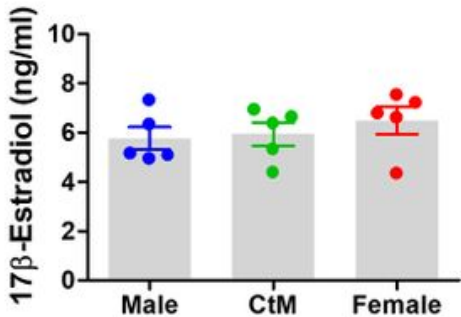

d
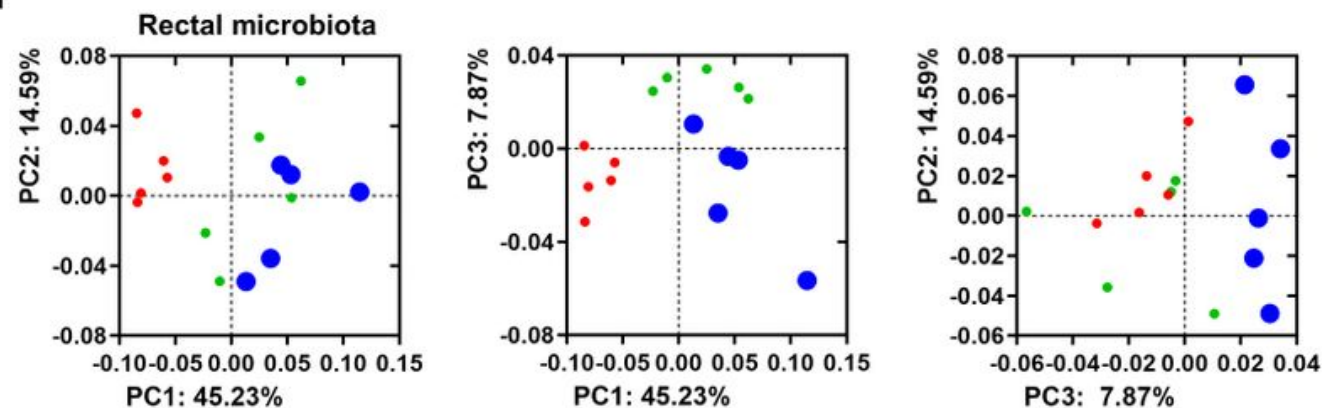

- Male

- CtM

Female

Testosterone ( $\mathrm{ng} / \mathrm{ml}$ )

PC1: $45.23 \%$

e

Serum metabolome
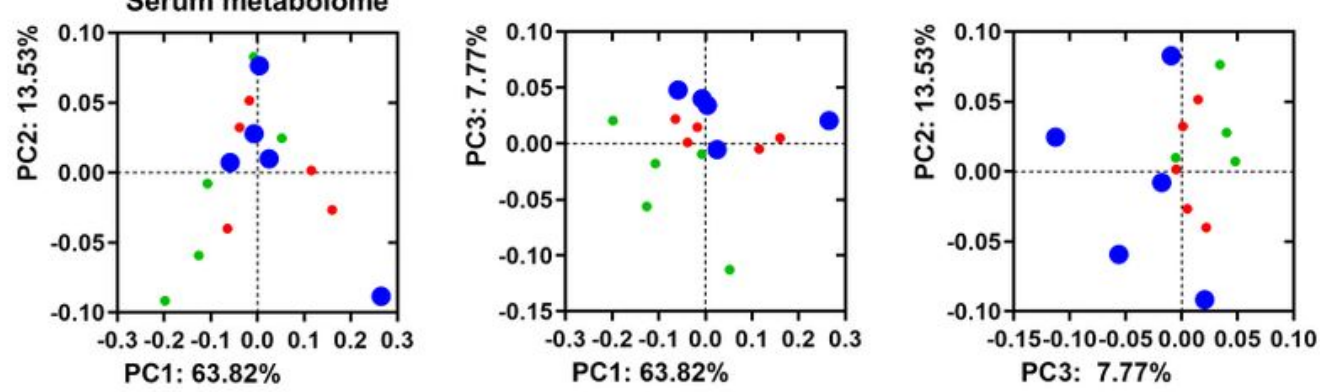

f

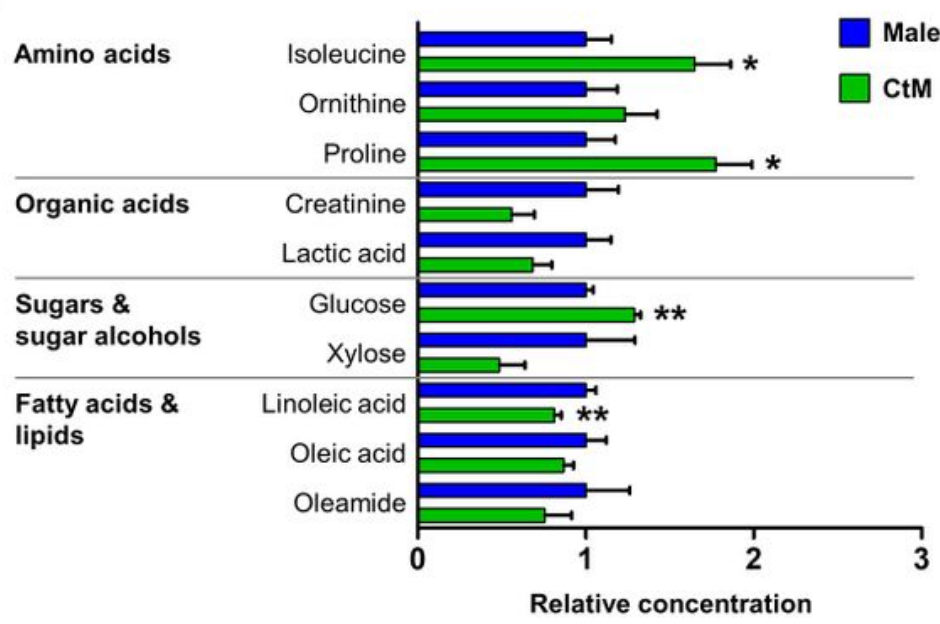

g

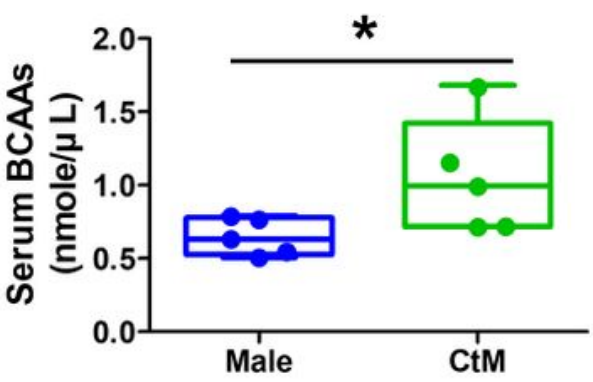

Figure 1

The rectal microbial and serum metabolome profiles of the postpubescent Hanwoo. a-c Determination of the body weight (a), and serum testosterone (b) and 17 $\beta$-estradiol (c) levels, in postpubescent male, castrated male (CtM), and female Hanwoo ( $n=5$ in each group). $d$ PCoA of the rectal bacterial 16S rRNA gene sequences based on the weighted UniFrac distance matrix. e The serum metabolome profiles were analyzed using GC-TOF-MS and clustered by PCoA based on the Bray-Curtis dissimilarity matrix. $f$ The 
relative abundances of serum metabolites displayed as bar graphs. $g$ The BCAA serum levels quantified enzymatically, as described in the materials and methods section. The data were analyzed by using ANOVA followed by Tukey's post-hoc test $(a-c)$ and the Mann-Whitney $U$ test ( $f$ and $g$ ). ${ }^{*} p<0.05,{ }^{* \star} p<0.01$, and $* * * p<0.001$. CtM, castrated male.

a

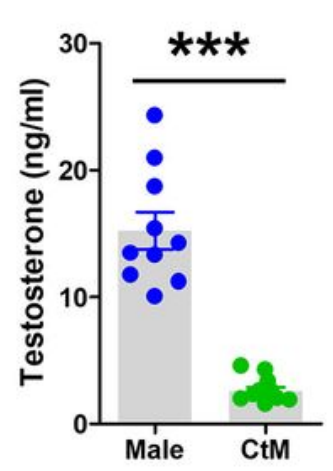

C

C Total microbiota (weighted UniFrac) b

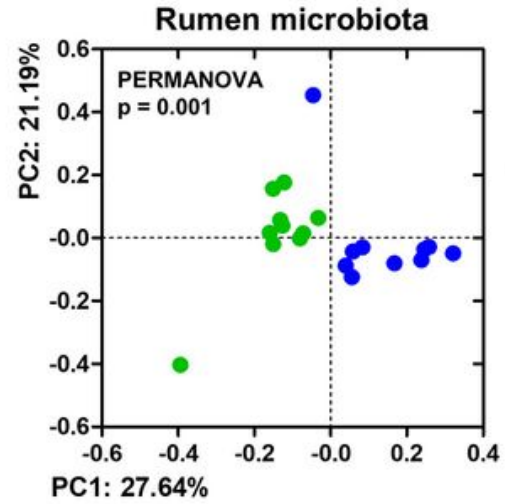

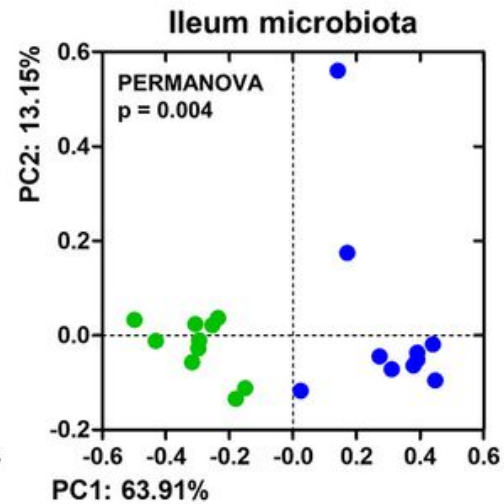

d

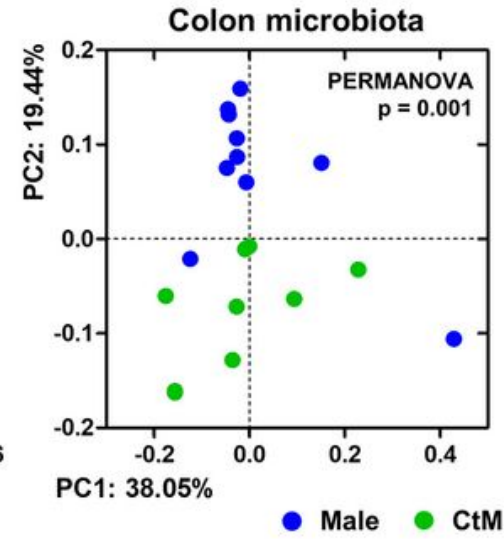

Distance between Male and CtM

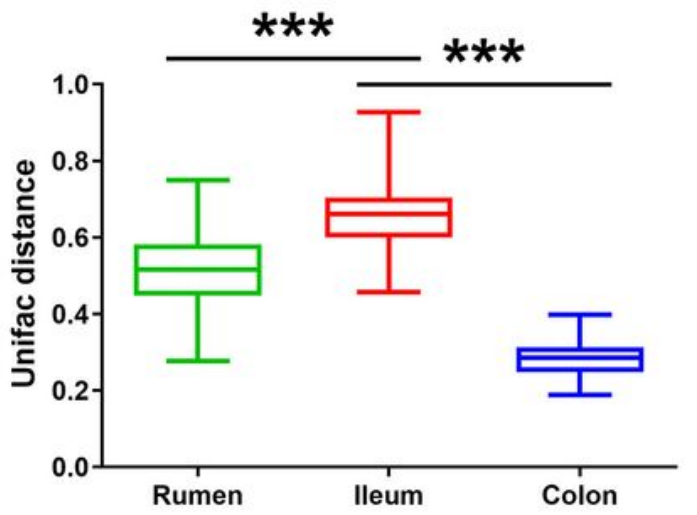

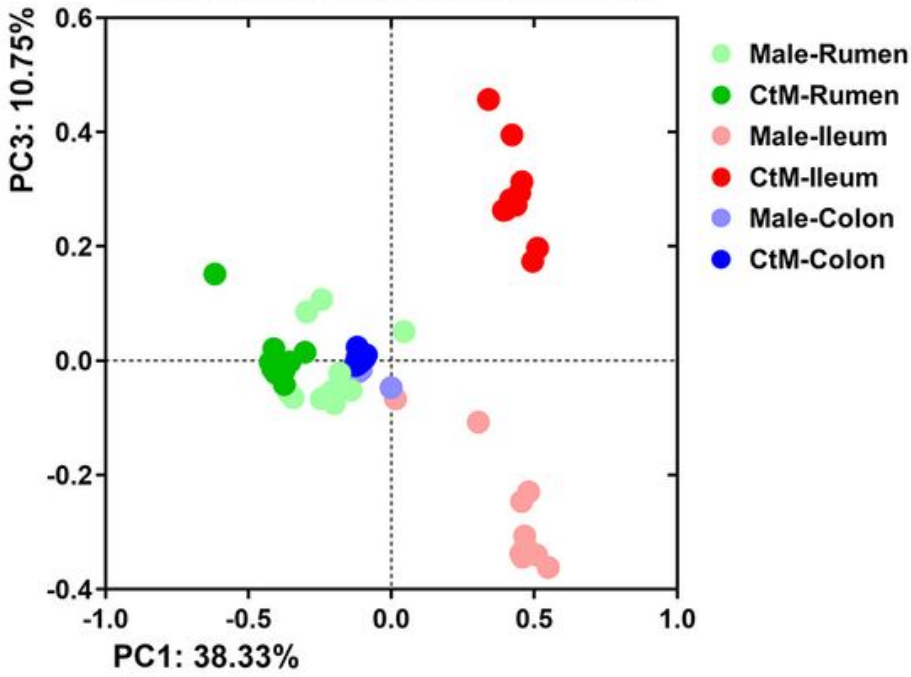

Unassigned

Sum of rare taxa Christensenellaceae - Planococcaceae - Coriobacteriaceae = Succinivibrionaceae = Spirochaetaceae nikenellaceae

= Erysipelotrichaceae Mogibacteriaceae = S24-7

= Turicibacteraceae - Verrucomicrobiaceae m F16

" Bacteroidaceae - Veillonellaceae n Paraprevotellaceae = Methanobacteriaceae - Clostridiaceae - Peptostreptococcaceae - Prevotellaceae

=Lachnospiraceae numinococcaceae

Figure 2 
Microbial metataxonomic profiles of different compartments of the intestinal tract of the adult male and castrated male (CtM) Hanwoo. a Serum testosterone levels in the adult male $(n=10)$ and CtM $(n=10)$ Hanwoo. b-c PCoA, based on the weighted UniFrac distance matrix, of the bacterial 16S rRNA gene sequence data for the rumen, ileum, and colon, shown for the different segments of the gastrointestinal tract (b) and combined data (c). d Microbial dissimilarity (calculated based on the weighted UniFrac distance matrix) between the male and CtM groups in the different intestinal compartments. e The relative abundances of abundant bacterial taxa $(>0.5 \%$ of the mean abundance). The data were analyzed by using the Mann-Whitney U test (a), PERMANOVA with 999 permutations (b), or ANOVA followed by Tukey's post-hoc test (d). ${ }^{*} p<0.05$, ${ }^{* *} p<0.01$, and ${ }^{* * *} p<0.001$. CtM, castrated male.

a
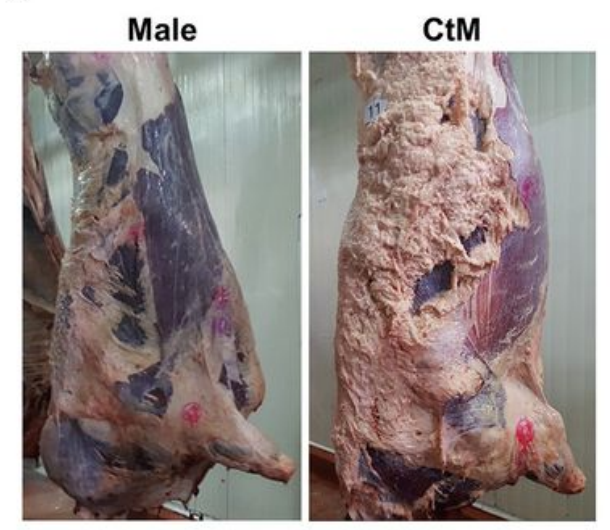

c
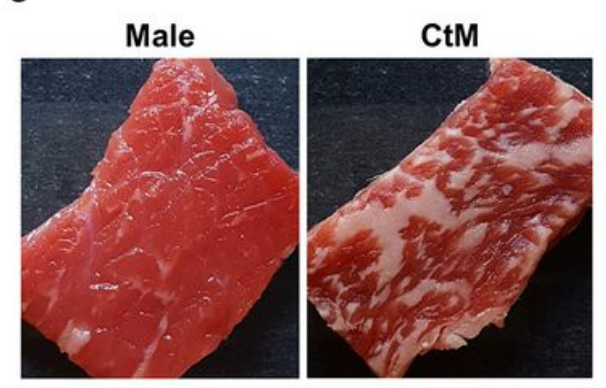

$f$
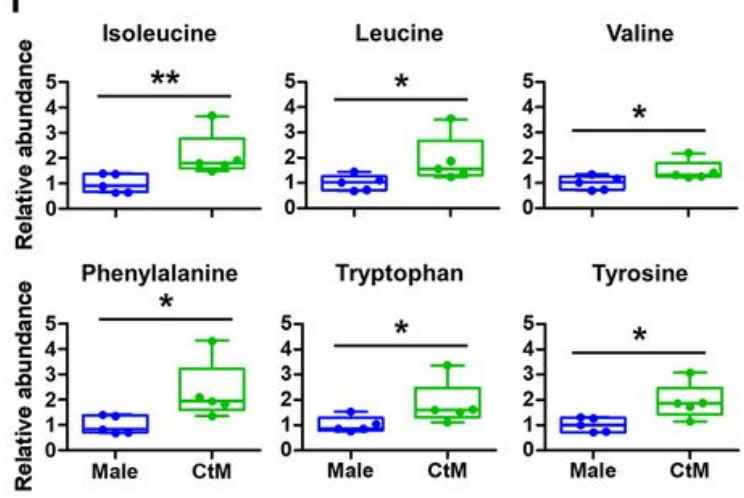

d

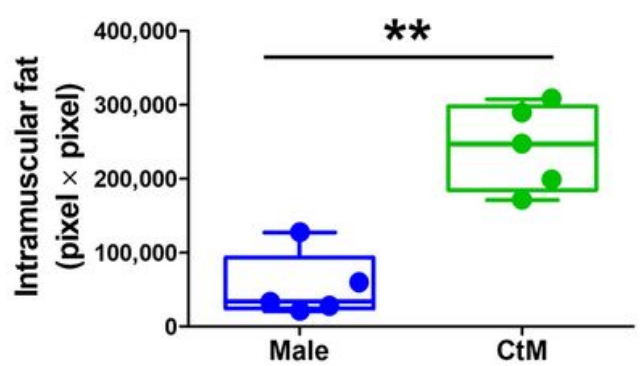

g

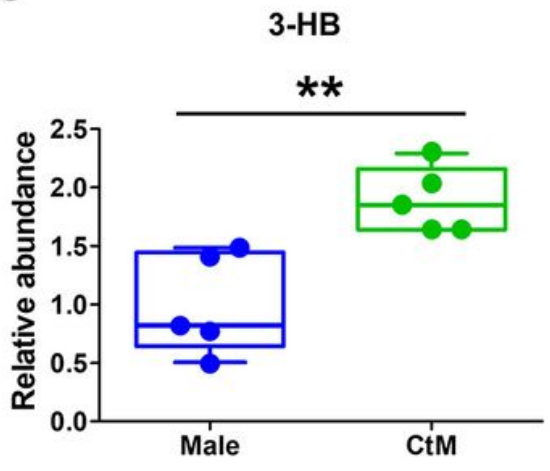

e

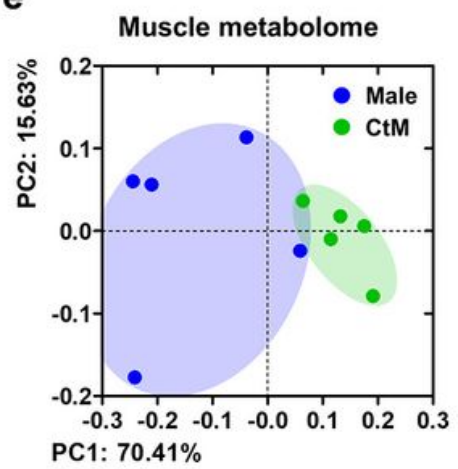

\section{Figure 3}


Intramuscular metabolome profiles of the adult male and castrated male (CtM) Hanwoo. a

Representative images of dressed bodies of the adult male and CtM Hanwoo ( $n=10$ in each group). $b$ Dressed body weight, thickness of the dorsal subcutaneous fat, total fat weight, and serum levels of the branched-chain amino acids (BCAAs). c Representative images of fresh striploin muscle from the adult male and CtM carcasses ( $n=5$ in each group). $d$ The intramuscular fat area in size-normalized muscle. $e$ The intramuscular metabolome profiles analyzed using GC-TOF-MS and clustered by PCoA based on the Bray-Curtis dissimilarity matrix. $f-g$ The relative abundances of intramuscular metabolites (f) and $\beta$ hydroxybutyrate (3-HB) $(\mathrm{g})$ in the male and CtM samples. The data were analyzed by using the MannWhitney $U$ test (b, $d, f$ and $g$ ). ${ }^{*} p<0.05$, ${ }^{\star *} p<0.01$, and ${ }^{* \star *} p<0.001$. CtM, castrated male; BCAAs, branchedchain amino acids; 3-HB, $\beta$-hydroxybutyrate. 
a

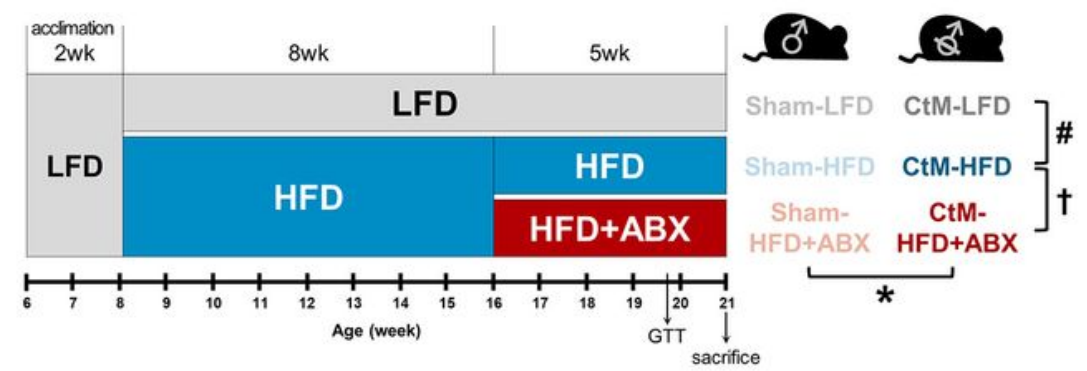

C

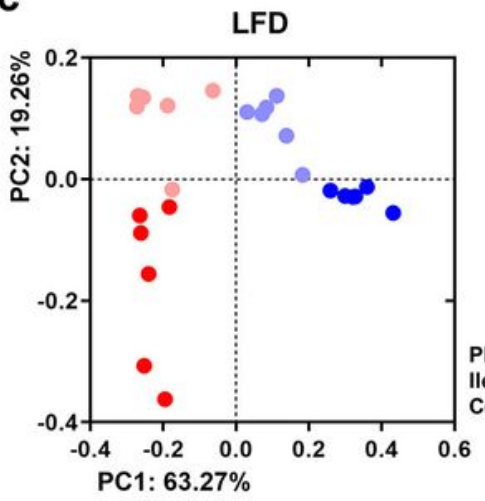

HFD

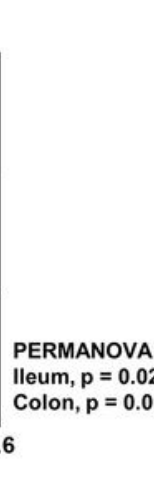

b
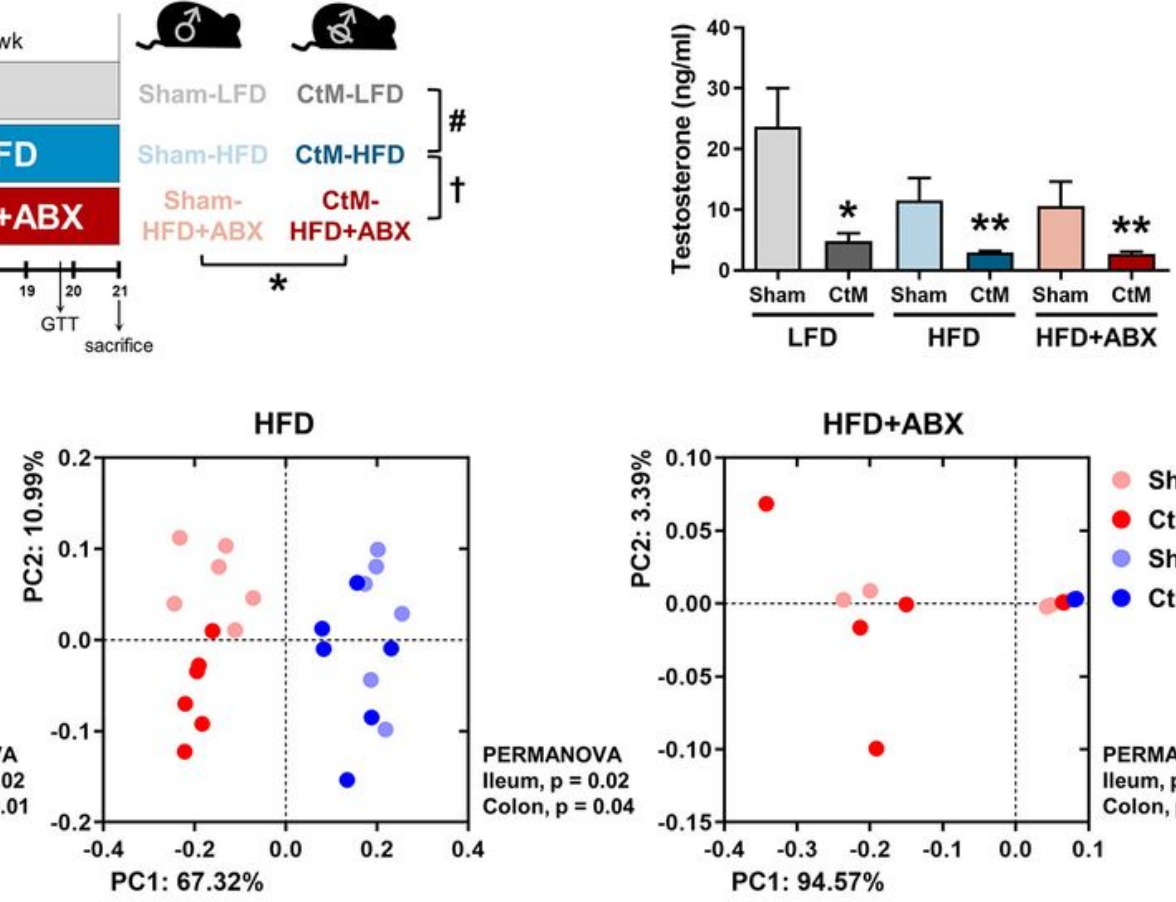

HFD+ABX

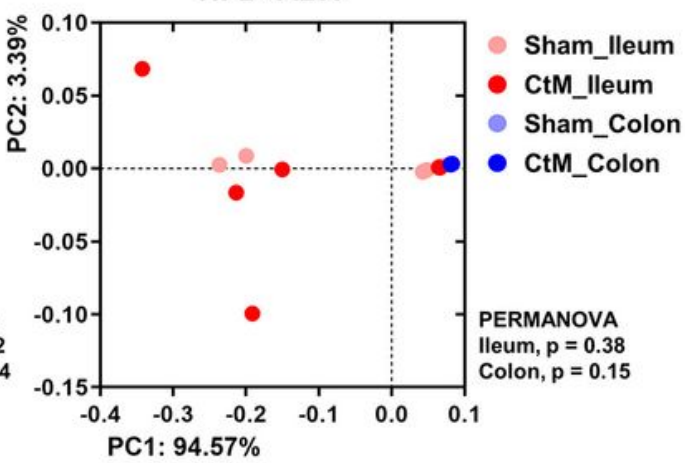

d

e

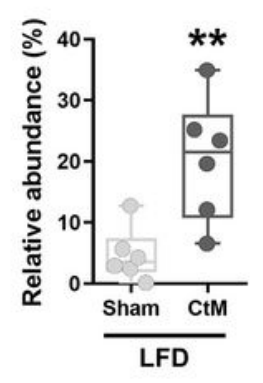

f
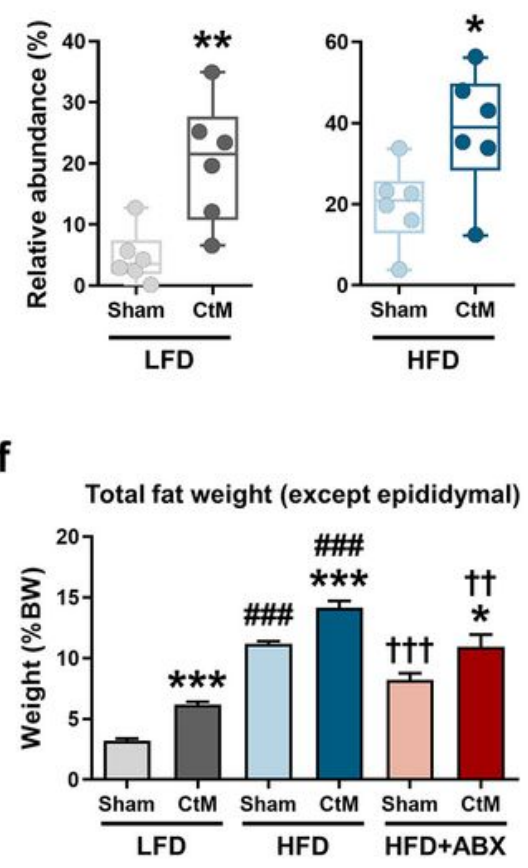

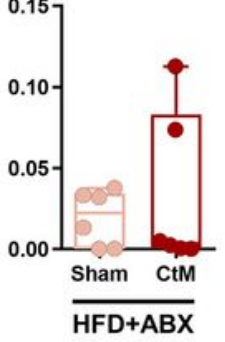

g

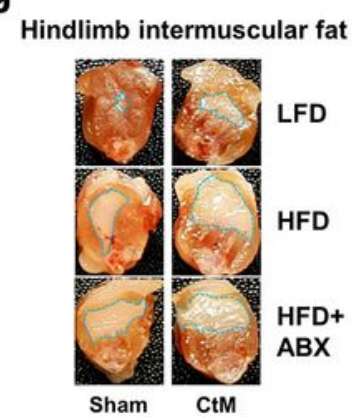

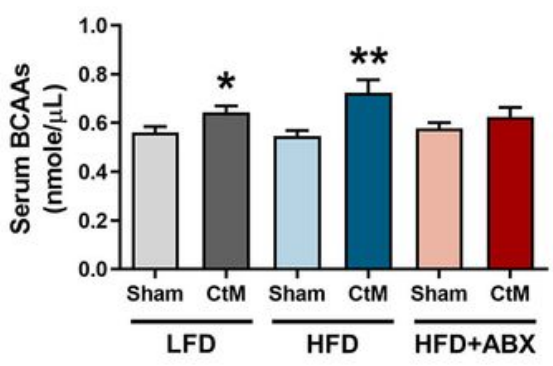

h

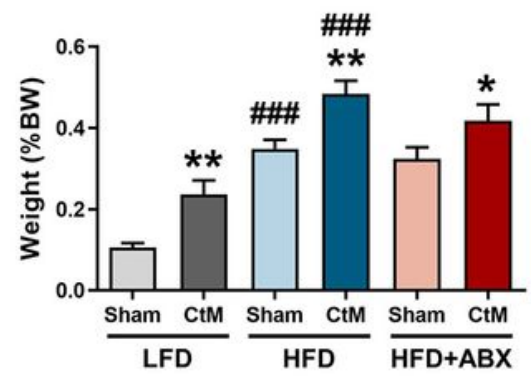

Figure 4

The intestinal microbiota and metabolic phenotypes of the male and castrated male (CtM) mice. a Schematic design of the hypogonadism experiments in the mouse model. b Serum testosterone levels. $c$ PCoA, based on the weighted UniFrac distance matrix, of the bacterial 16S rRNA gene sequence data for the luminal contents of the ileum and colon, shown according to the different diets and antibiotic treatment. $d$ The relative abundances of the family Peptostreptococcaceae are displayed as a box and 
dot plot. e The serum branched-chain amino acid (BCAA) levels quantified enzymatically, as described in the materials and methods section. $f$ The total weight of the extramuscular fat, including posterior subcutaneous fat, mesenteric fat, and retroperitoneal fat. $\mathrm{g}-\mathrm{h}$ Representative images $(\mathrm{g})$ and weight $(\mathrm{h})$ of the hindlimb intermuscular fat. The images are from a longitudinal section of the hind leg. The fat weight data are presented as a percentage of the body weight. The data were analyzed by using the unpaired Student's t-test ( $b, d-f$, and $h$ ) or PERMANOVA with 999 permutations (c). *Comparison of the Sham and CtM mice; \#comparison of the LFD and HFD mice; and tcomparison of the HFD and HFD+ABX mice. ${ }^{*} p<0.05,{ }^{\star} \times p<0.01$, and ${ }^{* \star *} p<0.001$. LFD, low-fat diet; HFD, high-fat diet; ABX, antibiotics; CtM, castrated male; BCAAs, branched-chain amino acids.

a

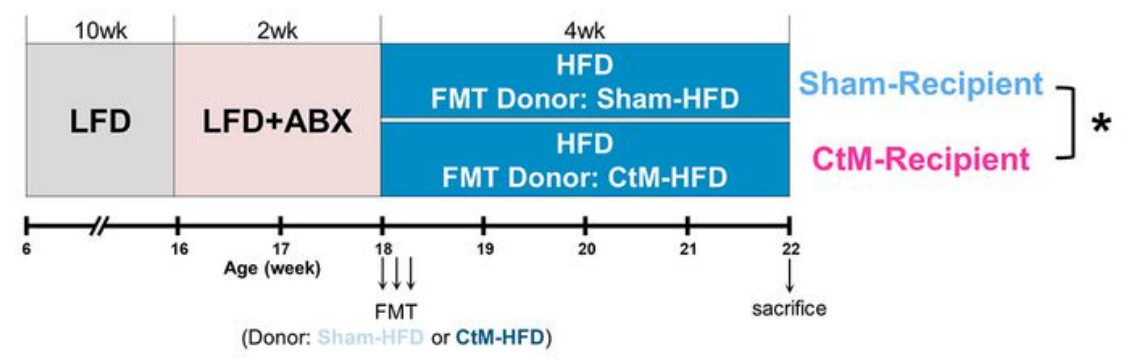

b

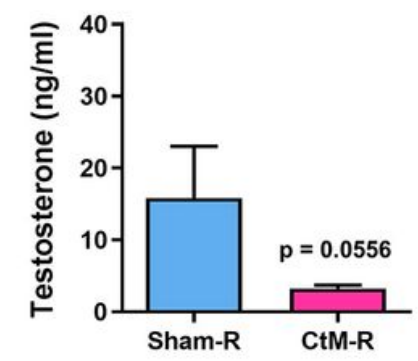

C

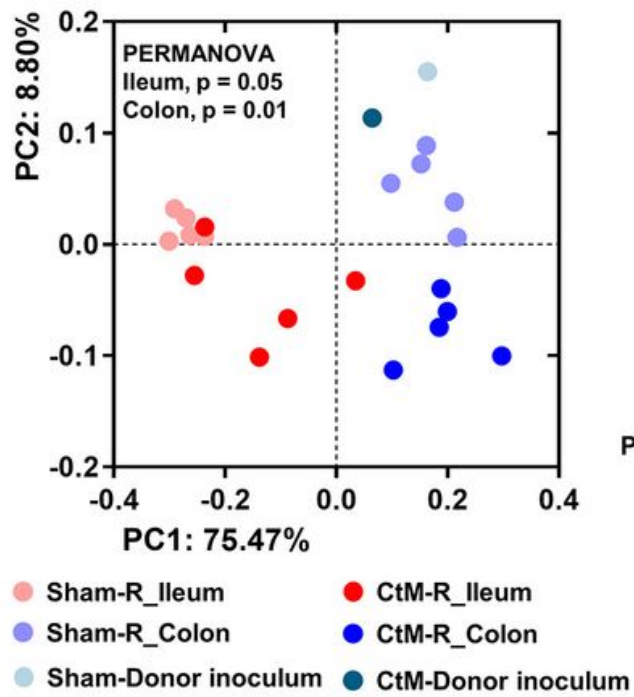

d

lleum microbiota

Sham-R $\square$ CtM-R

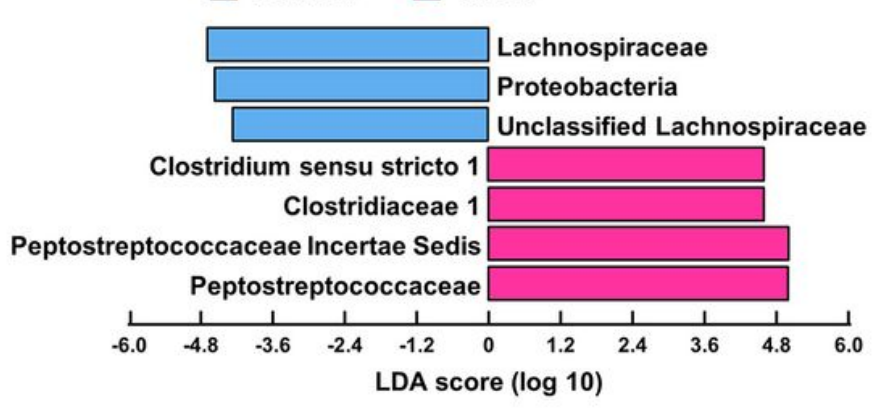

Lachnospiraceae

Proteobacteria

Unclassified Lachnospiraceae

ostridium sensu stricto 1

Clostridiaceae 1

Peptostrepto

LDA score $(\log 10)$ e

Ileum Peptostreptococcaceae

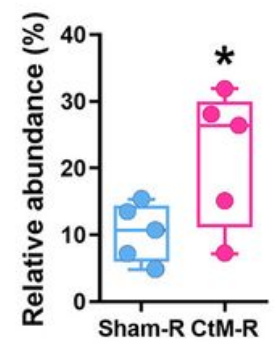

f

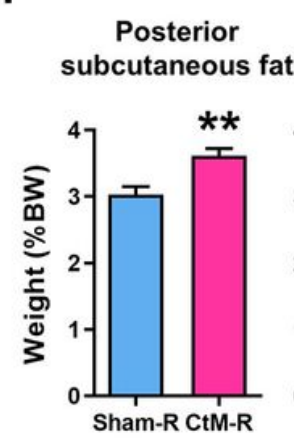
$\underset{\text { fat }}{\text { Epididymal }}$

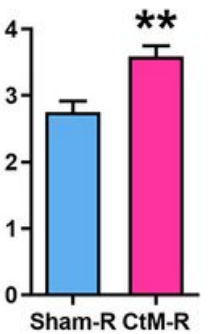

Mesenteric fat

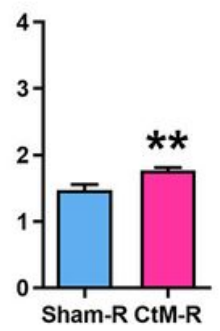

Retroperitoneal fat

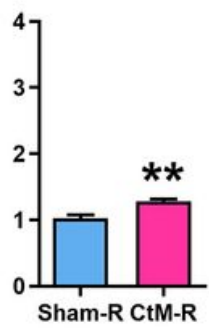

g

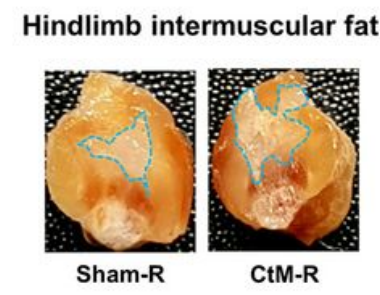

h

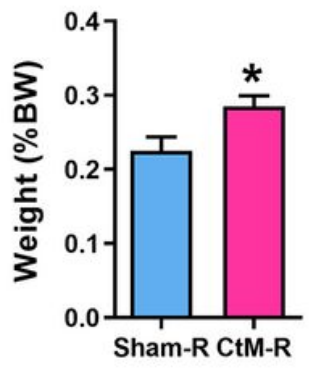

Figure 5 
The intestinal microbiota and metabolic phenotypes of male mice in response to fecal microbiota transplantation (FMT). a Schematic design for the mouse FMT experiments. $b$ The serum testosterone levels in the Sham-R and castrated male (CtM)-R mice. c PCoA, based on the weighted UniFrac distance matrix, of the bacterial 16S rRNA gene sequence data for the luminal contents of the ileum and colon. $d$ The discriminant microbial taxa were determined by using the LEfSe and presented using the LDA score. e The relative abundances of the family Peptostreptococcaceae are displayed as a box and dot plot. $f$ Weights of the posterior subcutaneous fat, epididymal fat, mesenteric fat, and retroperitoneal fat in the Sham-R and CtM-R mice. $\mathrm{g}-\mathrm{h}$ Representative images $(\mathrm{g})$ and weight $(\mathrm{h})$ of the hindlimb intermuscular fat in the Sham-R and CtM-R mice. Images were obtained from a longitudinal section of the hind leg. The fat weight data are presented as a percentage of the body weight. The data were analyzed by using the unpaired Student's t-test ( $b, e, f$, and $h$ ) or PERMANOVA with 999 permutations $(c) .{ }^{*} p<0.05$ and ${ }^{* *} p<0.01$. LFD, low-fat diet; HFD, high-fat diet; ABX, antibiotics; FMT, fecal microbiota transplantation; CtM, castrated male. 


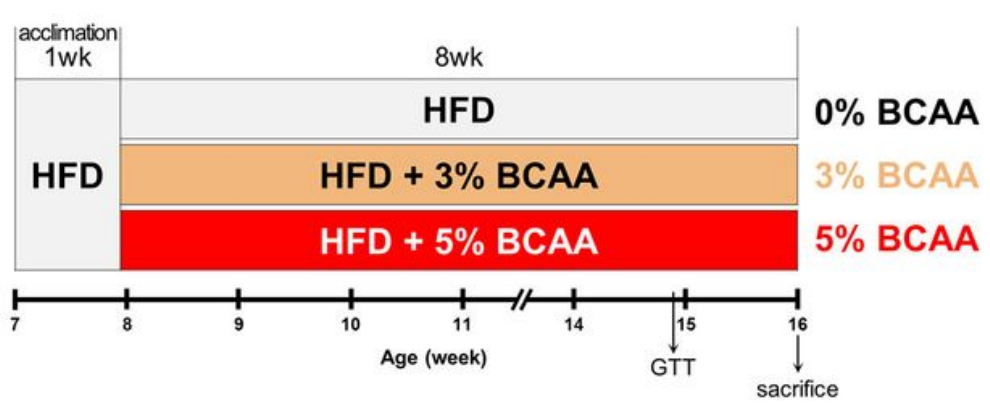

b

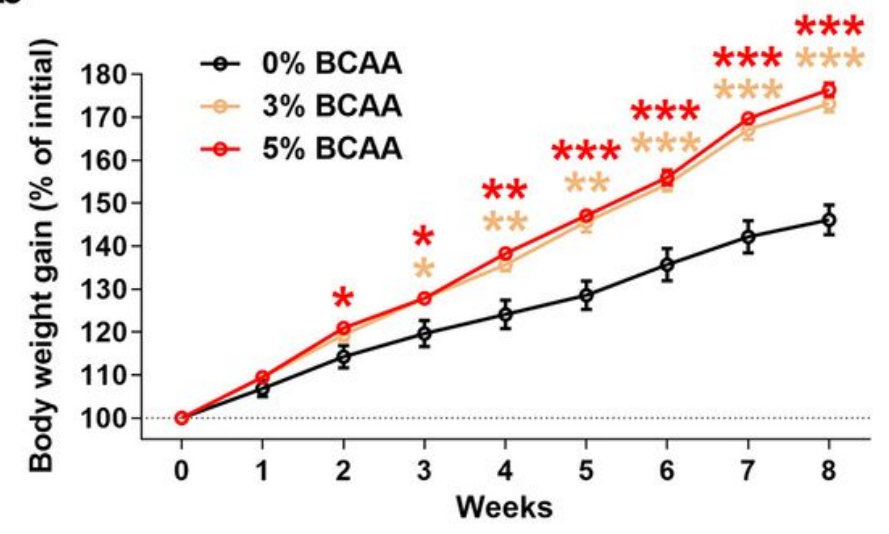

e

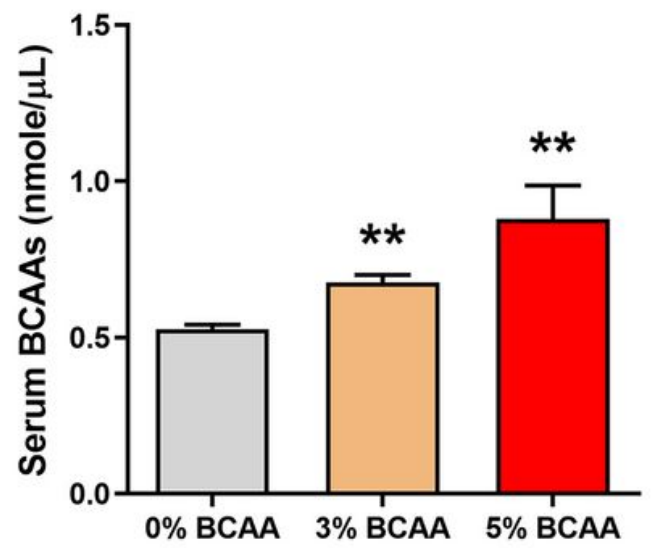

C

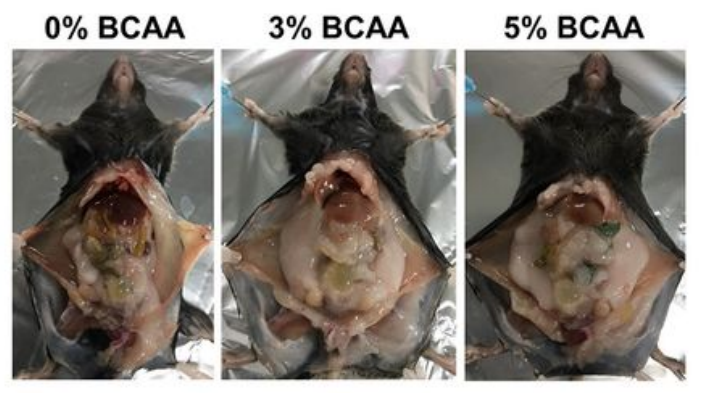

d

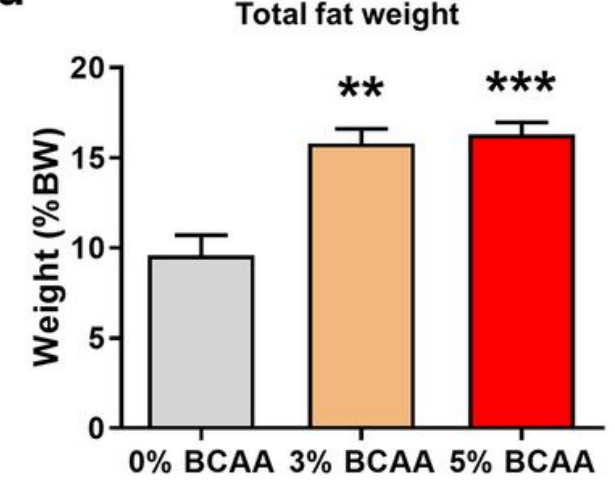

f

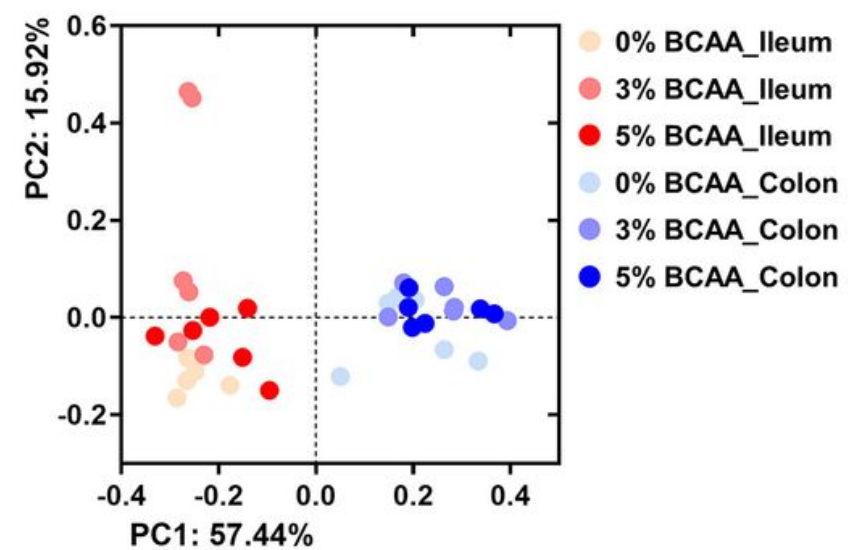

Figure 6

The metabolic phenotypes and intestinal microbiota of male mice fed branched-chain amino acid (BCAA)-supplemented diets. a Schematic design of the chronic BCAA feeding experiments. b Body weight gain in response to the different levels of dietary BCAAs. c Representative images of gross anatomy. $d$ The total weight of the extramuscular fat (posterior subcutaneous fat, epididymal fat, mesenteric fat, and retroperitoneal fat) in the mice fed high-fat diet (HFD) with or without BCAAs. e The serum BCAA levels in the mice fed HFD with or without BCAAs. $\mathrm{PCoA}$, based on the weighted UniFrac distance matrix, of the bacterial 16S rRNA gene sequence data for the luminal contents of the ileum and colon are shown. The body weight gain data are presented as a percentage of the initial body weight. The fat weight data are presented as a percentage of the body weight. The data were analyzed by using the unpaired Student's t- 
test (b, d, and e). ${ }^{*}$ Comparison of the $0 \%$ BCAA and $3 \%$ BCAA or $5 \%$ BCAA-fed mice. ${ }^{*} p<0.05,{ }^{*} p<<0.01$, and ${ }^{\star * \star} p<0.001$. HFD, high-fat diet; BCAA, branched-chain amino acid.

\section{Supplementary Files}

This is a list of supplementary files associated with this preprint. Click to download.

- Additionalfile1SuppleFigs.pdf

- Additionalfile3Suppleresults.pdf

- Additionalfile2SuppleTables.pdf 